\title{
THE SPITZER c2d SURVEY OF LARGE, NEARBY, INTERSTELLAR CLOUDS. IX. THE SERPENS YSO POPULATION AS OBSERVED WITH IRAC AND MIPS
}

\author{
Paul Harvey, ${ }^{1}$ Bruno Merín, ${ }^{2}$ Tracy L. Huard, ${ }^{3}$ Luisa M. Rebull, ${ }^{4}$ Nicholas Chapman, ${ }^{5}$ \\ Neal J. Evans II, ${ }^{1}$ and Philip C. Myers ${ }^{3}$ \\ Received 2007 January 29; accepted 2007 April 1
}

\begin{abstract}
We discuss the combined IRAC/MIPS c2d Spitzer Legacy observations of the Serpens star-forming region. We describe criteria for isolating bona fide YSOs from the extensive background of extragalactic objects. We then discuss the properties of the resulting high-confidence set of 235 YSOs. An additional 51 lower confidence YSOs outside this area are identified from the MIPS data and 2MASS photometry. We present color-color diagrams to compare our observed source properties with those of theoretical models for star/disk/envelope systems and our own modeling of the objects that are well represented by a stellar photosphere plus circumstellar disk. These objects exhibit a wide range of disk properties, from many with actively accreting disks to some with both passive disks and even possibly debris disks. The YSO luminosity function extends down to at least a few times $10^{-3} L_{\odot}$ or lower. The lower limit may be set more by our inability to distinguish YSOs from extragalactic sources than by the lack of YSOs at very low luminosities. We find no evidence for variability in the shorter IRAC bands between the two epochs of our data set, $\Delta t \sim 6 \mathrm{hr}$. A spatial clustering analysis shows that the nominally less evolved YSOs are more highly clustered than the later stages. The background extragalactic population can be fitted by the same two-point correlation function as seen in other extragalactic studies. We present a table of matches between several previous infrared and X-ray studies of the Serpens YSO population and our Spitzer data set. The clusters in Serpens have a very high surface density of YSOs, primarily with SEDs suggesting extreme youth. The total number of YSOs, mostly Class II, is greater outside the clusters.
\end{abstract}

Subject headings: infrared: general

Online material: machine-readable tables

\section{INTRODUCTION}

The Serpens star-forming cloud is one offive such large clouds selected for observation as part of The Spitzer Legacy project "From Molecular Cores to Planet-forming Disks" (c2d; Evans et al. 2003). Previous papers in this series have described the observational results in the Serpens cloud as seen with IRAC (Harvey et al. 2006, hereafter Paper II) and MIPS (Harvey et al. 2007), as well as some of the other clouds (Jorgensen et al. 2006; Rebull et al. 2007). In this paper we examine how the combination of the IRAC and MIPS data together with other published results on this region can be used to find and characterize a highly reliable catalog of young stellar objects (YSOs) in the surveyed area. With the combination of broad wavelength coverage and amazing depth of Spitzer's sensitivity we are able both to probe to extremely low luminosity limits for YSOs and to cover a very wide range in dust emission, both in optical depth and in range of emitting temperatures. The Spitzer wavelength region is particularly well tuned for sensitivity to dust at temperatures appropriate for solar system-size disks around young stars.

The region of the Serpens cloud mapped in our survey is an area rich in star formation. Eiroa et al. (2007) have extensively reviewed studies at a variety of wavelengths of this area. There is

\footnotetext{
1 Astronomy Department, University of Texas at Austin, Austin, TX 787120259; pmh@astro.as.utexas.edu,nje@astro.as.utexas.edu.

2 Research and Scientific Support Department, ESTEC (ESA), 2200 AG Noordwijk, Netherlands; and Leiden Observatory, Leiden University, 2300 RA Leiden, Netherlands; bmerin@rssd.esa.int.

3 Smithsonian Astrophysical Observatory, Cambridge, MA 02138; thuard@ cfa.harvard.edu, pmyers@cfa.harvard.edu.

4 Spitzer Science Center, Pasadena, CA 91125; rebull@ipac.caltech.edu.

5 Astronomy Department, University of Maryland, College Park, MD 20742; chapman@astro.umd.edu.
}

evidence from previous observations of strong clustering (Testi et al. 2000; Testi \& Sargent 1998), dense submillimeter cores (Casali et al. 1993; Enoch et al. 2006b), and high-velocity outflows (Ziener \& Eisloffel 1999; Davis et al. 1999). Pre-Spitzer infrared surveys of the cloud have been made by IRAS (Zhang et al. 1988a, 1988b) and the Infrared Space Observatory (ISO; Kaas et al. 2004; Djupvik et al. 2006), as well as the pioneering ground-based surveys that first identified it as an important region of star formation (Strom et al. 1976). Using MIPS and all four bands of IRAC, the c2d program has mapped a $0.85 \mathrm{deg}^{2}$ portion of this cloud that includes a very well studied cluster of infrared and submillimeter sources (Eiroa \& Casali 1992; Hogerheijde et al. 1999; Hurt \& Barsony 1996; Harvey et al. 1984; Testi \& Sargent 1998). At its distance of $260 \pm 10 \mathrm{pc}$ (Straizys et al. 1996) this corresponds to an area of about $2.5 \times 9 \mathrm{pc}$. In Paper II we identified at least two main centers of star formation as seen by Spitzer in this cloud, which we referred to as clusters A and B. Cluster A is the very well studied grouping also commonly referred to as the Serpens core. Cluster B was the subject of a recent multiwavelength study by Djupvik et al. (2006), who referred to it as the Serpens G3-G6 cluster.

The 235 YSOs with high signal-to-noise ratio $(\mathrm{S} / \mathrm{N})$ that we have cataloged constitute a sufficiently large number that we can examine statistically the numbers of objects in various evolutionary states and the range of disk properties for different classes of YSOs. We characterize the circumstellar material with colorcolor diagrams that allow comparison with other recent studies of star-forming regions, and we model the energy distributions of the large number of YSOs that appear to be star+circumstellar disk systems. We are able to construct the YSO luminosity function for Serpens since we have complete spectral coverage for all the sources over the range of wavelengths where their luminosity 
is emitted, and we are able to characterize the selection effects inherent in the luminosity function from comparison with the publicly available and significantly deeper SWIRE survey (Surace et al. $2004^{6}$ ). We find that the population of YSOs extends down to luminosities below $10^{-2} L_{\odot}$, and we discuss the significance of this population. Our complete coverage in wavelength and luminosity space also permits us to discuss the spatial distribution of YSOs to an unprecedented completeness level. We note also that, unlike the situation discussed by Jorgensen et al. (2006) for Perseus, in Serpens there do not appear to be any very deeply embedded YSOs that are not found by our YSO selection criteria.

In $\S 2$ we briefly review the observational details of this program, and then in $\S 3$ we describe in detail the process by which we identify YSOs and eliminate background contaminants. In $\S 4$ we describe a search for variability in our data set. We compare our general results on YSOs with those of earlier studies of the Serpens star-forming region in $\S 5$. We discuss in $\S 6$ the YSO luminosity function in Serpens and, in particular, the low end of this function. We next analyze the spatial distribution of star formation in the surveyed area and compare it to the distribution of dust extinction as derived from our observations in $\S 7$. In $\S 8$ we construct several color-color diagrams that characterize the global properties of the circumstellar material and show modeling results for a large fraction of our YSOs that appear to be star+disk systems. Finally, $\S 9$ discusses several specific groups of objects including the coldest YSOs and a previously identified "disappearing" YSO. We also mention several obvious high-velocity outflows from YSOs that will certainly be the subject of further study.

\section{OBSERVATIONS}

The parameters of our observations have already been described in detail in Paper II for IRAC and in a companion study (Harvey et al. 2007) for MIPS. We summarize here some of the issues most relevant to this study.

We remind the reader that the angular resolution of the Spitzer imaging instruments varies widely with wavelength, since Spitzer is diffraction limited longward of $\lambda \sim 10 \mu \mathrm{m}$. In the shorter IRAC bands the spatial resolution is of order $2^{\prime \prime}$, while from 24 to $160 \mu \mathrm{m}$, it goes from roughly $5^{\prime \prime}$ to $50^{\prime \prime}$. The area chosen for mapping was defined by the $A_{V}>6$ contour in the extinction map of Cambrésy (1999) and by practical time constraints (Evans et al. 2003). With the exception of a small area of $0.04 \mathrm{deg}^{2}$ on the northeast edge of the IRAC map, all of the area mapped with IRAC was also covered with MIPS at both 24 and $70 \mu \mathrm{m}$, and most of it at $160 \mu \mathrm{m}$. As described by Harvey et al. (2007), some substantial additional area was observed with MIPS without matching IRAC observations. In this paper we restrict our attention to only the area that was observed from 3.6 to $70 \mu \mathrm{m}, 0.85 \mathrm{deg}^{2}$. This entire region was observed from 3.6 to $24 \mu \mathrm{m}$ at two epochs, with a time separation of several hours to several days, but at only one epoch at $70 \mu \mathrm{m}$. We also specifically do not include the $160 \mu \mathrm{m}$ observations in our discussion because they did not cover the entire area and because most of the YSOs in Serpens are too closely clustered to be distinguishable in the large beam of the $160 \mu \mathrm{m}$ data. Harvey et al. (2007) discuss briefly the extended $160 \mu \mathrm{m}$ emission in this region and the four pointlike sources found at $160 \mu \mathrm{m}$. Figure 1 shows the entire area mapped with all four IRAC bands and MIPS at 24 and $70 \mu \mathrm{m}$ and also indicates the locations of several areas mentioned in the text.

\footnotetext{
6 Available at http://ssc.spitzer.caltech.edu/legacy/.
}

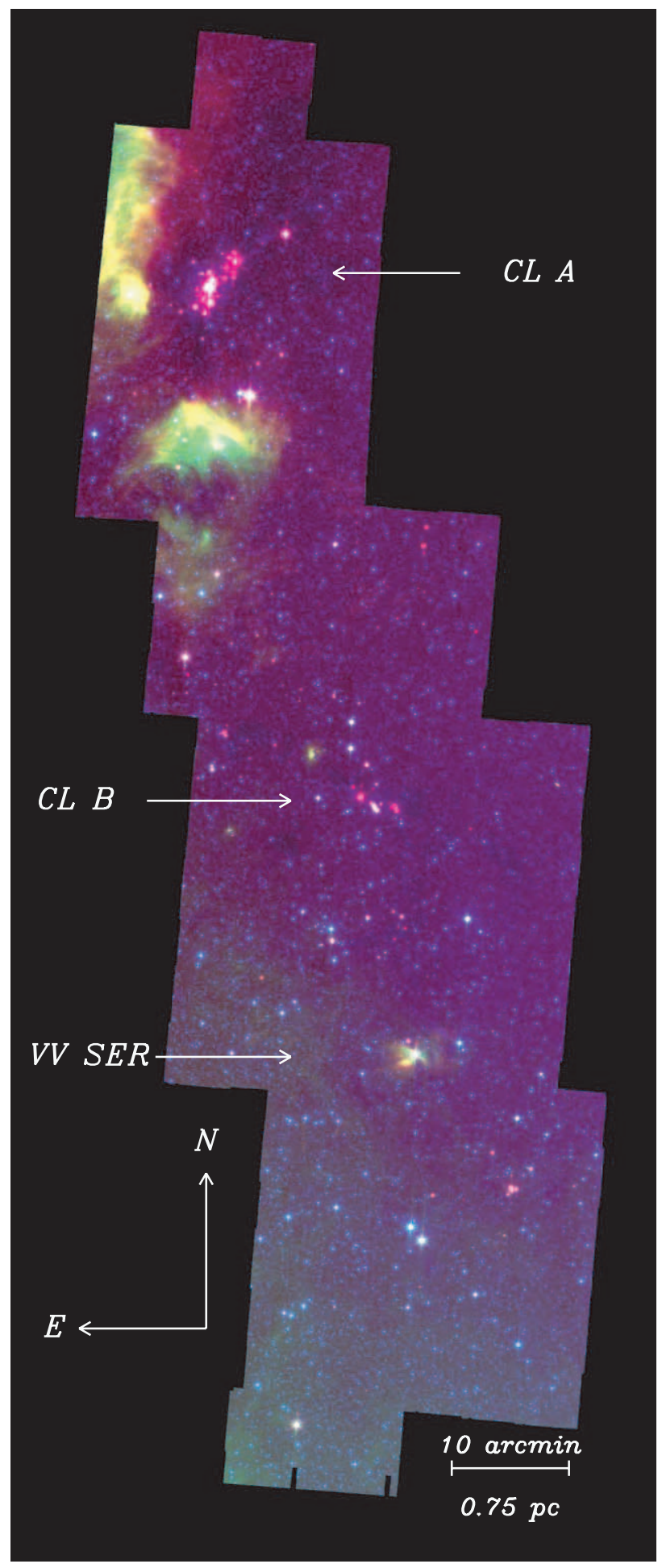

FIG. 1.- Three-color image of the area mapped in Serpens. The color mapping is blue, green, and red for $4.5,8.0$, and $24 \mu \mathrm{m}$, respectively. The locations of cluster A (core cluster), cluster B (Serpens G3-G6 cluster), and VV Ser are indicated.

In addition to this area of the Serpens cloud defined by relatively high $A_{V}$, we also observed small off-cloud regions around the molecular cloud with relatively low $A_{V}$ in order to determine the background star and galaxy counts. The area of combined IRAC/MIPS coverage of these off-cloud regions, however, was relatively small and so these observations are not discussed further in this paper. As detailed below, we have used the much 
larger and deeper SWIRE (Surace et al. 2004) survey to understand the characteristics of the most serious background contaminants in our maps, the extragalactic objects.

\section{YSO SELECTION}

Paper II described a process for classifying infrared objects into several categories: those whose energy distributions could be well fitted as reddened stellar photospheres, those that had a high likelihood of being background galaxies, and those that were viable YSO candidates. We have refined this process by combining MIPS and IRAC data together, as well as by producing an improved comparison catalog from the SWIRE (Surace et al. 2004) survey, trimmed as accurately as possible to the c2d sensitivity limits. As shown by the number counts versus Wainscoat models of the Galactic background toward Serpens in Paper II, nearly all the sources observed in our survey are likely to be background stars. Because of the high sensitivity of IRAC channels 1 and 2 relative to both the Two Micron All Sky Survey (2MASS) and IRAC channels 3 and 4, most of the more than 200,000 sources extracted from our Serpens data set do not have enough spectral coverage for any reliable classification algorithm. In particular, "only" 34,000 sources had enough spectral information from a combination of 2MASS and IRAC data to permit a test for consistency with a stellar photosphere plus extinction model, and nearly 32,000 of these objects were classified as reddened stellar photospheres. These normal stellar objects are not considered further in our discussion and are not plotted on the various color and magnitude diagrams. The details of this classification process and the criteria for fitting are described in detail by Evans et al. (2007).

In order to pursue the classification process for YSOs beyond that described in Paper II, we added one more step to the data processing described there. This final step was "band filling" the catalog to obtain upper limits or low-S/N detections of objects that were not found in the original source extraction processing. This step is described in detail in the delivery documentation for the final c2d data delivery (Evans et al. 2007). In short, however, it involved fixing the position of the source during an extraction at the position of an existing catalog source and fitting the image data at that fixed position for two parameters, a background level and source flux, assuming that it was a point source. For this processing step, the fluxes of all the originally extracted sources were subtracted from the image first. In the case of the data discussed in this paper, the most important contribution of this bandfilling step is to give us flux estimates for the YSO candidates and extragalactic candidates at $24 \mu \mathrm{m}$. Because our knowledge of the true point-spread function (PSF) is imperfect and because the $24 \mu \mathrm{m}$ PSF is so much larger than the IRAC ones, it was also necessary to examine these results carefully to be sure a bandfilled $24 \mu \mathrm{m}$ flux was not simply the poorly subtracted wings of a nearby bright source that, in fact, completely masked the source being band filled. For the purposes of this paper, all upper limits are given as $5 \sigma$ values.

Three of the sources eventually selected as YSOs by the process described below had $24 \mu \mathrm{m}$ fluxes that were obviously saturated. These are the objects in Table 2 below numbered 127, 137 , and 182. For these three objects we derived the $24 \mu \mathrm{m}$ flux from a fit to the wings of the source profiles, rather than a fit to the whole profile as is done by the standard $\mathrm{c} 2 \mathrm{~d}$ source extraction.

\subsection{Constructing a Control Catalog from Deep Extragalactic SWIRE Observations}

We use the IRAC and MIPS images of the ELAIS N1 field obtained by the SWIRE team as a control field for understanding

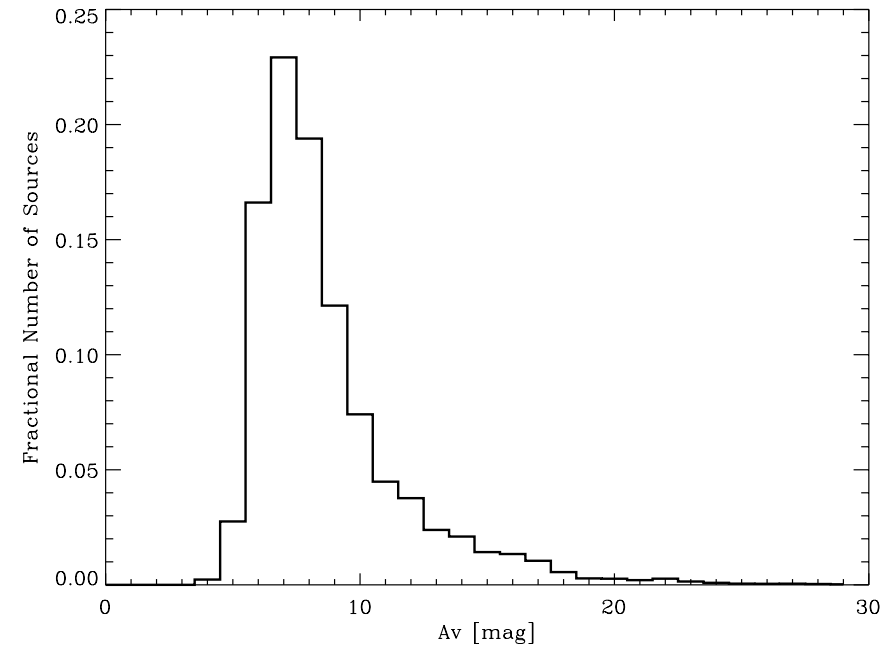

FIG. 2.-Distribution of visual extinctions found toward the roughly 50,000 sources classified as stars in our Serpens observations.

the extragalactic population with colors that mimic those of YSOs present in our Serpens field. This SWIRE field, expected to contain no extinction from a molecular cloud and no YSOs, has coverage by both IRAC and MIPS of $5.31 \mathrm{deg}^{2}$ and has a limiting flux roughly a factor of 4 below that of our observations of Serpens. The analysis described below is designed to produce a resampled version of the SWIRE field as it would have been observed with the typical c2d sensitivity and as if it were located behind a molecular cloud with the range of extinctions observed in Serpens. The process of simulating these effects is discussed in detail in the final c2d data delivery documentation (Evans et al. 2007), but the steps are summarized here.

To avoid effects that may result from differences in data processing, the BCD images for this SWIRE field were processed by our pipeline in exactly the same way as our own observations. Once a band-merged catalog of SWIRE sources was constructed, we first simulated the reddening of sources that would occur if Serpens had been in the foreground of this field. This reddening was accomplished by randomly applying extinction to each SWIRE source according to the extinction profile of Serpens, shown in Figure 2. For example, $\sim 23 \%$ of SWIRE sources were randomly selected and visual extinctions in the range $6.5 \leq A_{V}<$ 7.5 were applied, $\sim 19 \%$ of sources were extincted by extinctions in the range $7.5 \leq A_{V}<8.5$, and so forth. The extinctions were applied to each of the infrared bands according to the extinction law appropriate for molecular clouds and cores (T. L. Huard et al. 2007 , in preparation).

Second, we degraded the sensitivity of the reddened SWIRE photometry to match that of our Serpens observations. This was accomplished by matching the detection rates as a function of magnitude in each of the bands. The $90 \%$ completeness limits of the Serpens observations are approximately 16.6, 15.6, 15.0, 16.6, 16.2, 15.2, 13.4, and 9.6 mag at $J, H, K$, [3.6], [4.5], [5.8], [8.0], and [24], respectively. Thus, for each band, all reddened SWIRE sources brighter than the completeness limit in Serpens would be detectable by c2d-like observations and are identified as such in the resampled SWIRE catalog. Most, but not all, sources fainter than the completeness limit will not be detected by c2d-like observations. We randomly select which sources to identify as detections, in a given band, in such a way as to reproduce the empirically determined shape of the completeness function. This resampling process is performed for each band, resulting in those sources fainter than the completeness limits in some bands being 

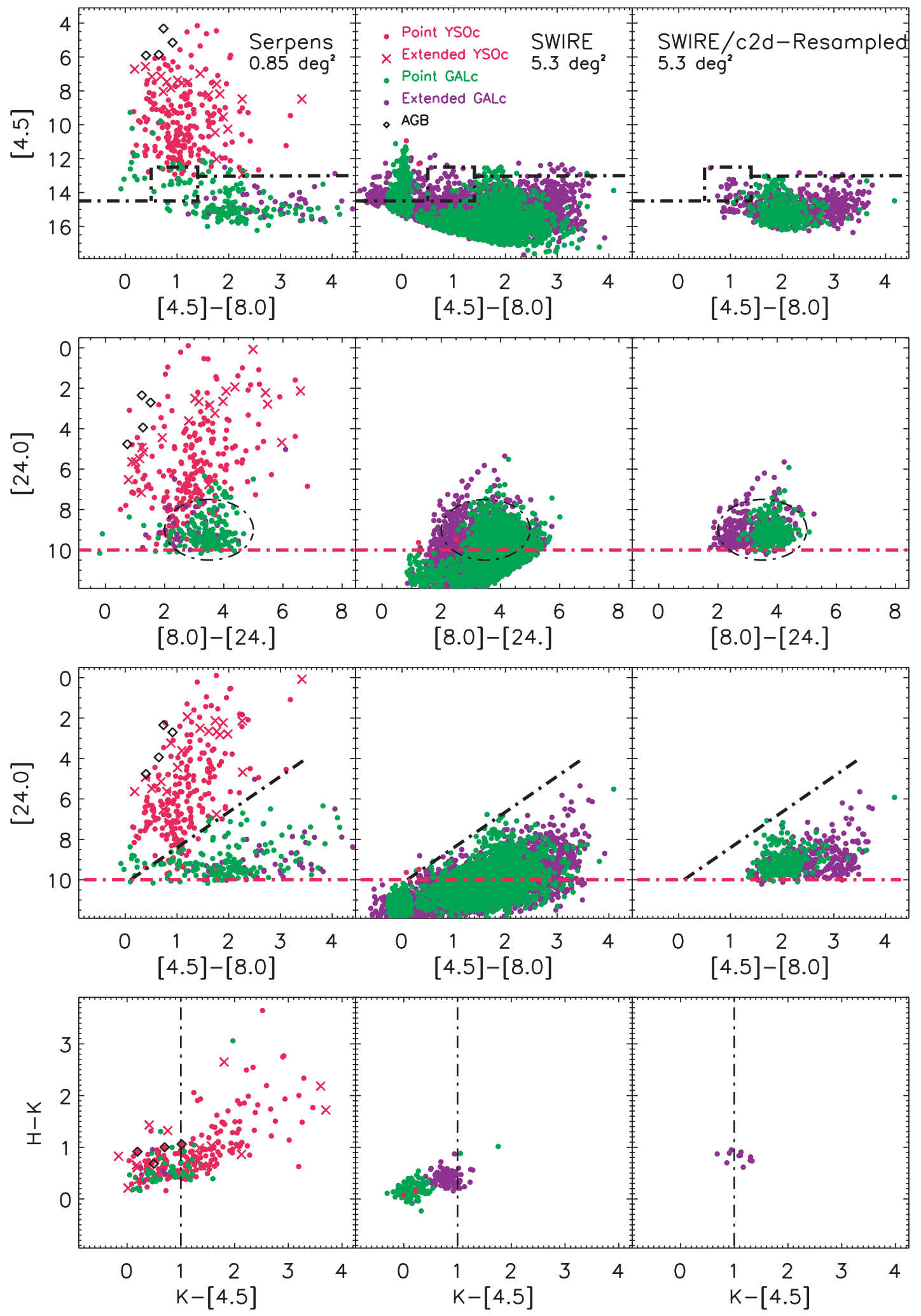

FIG. 3.-Color-magnitude and color-color diagrams for the Serpens cloud (left), full SWIRE (middle), and trimmed SWIRE (right) regions. The black dot-dashed lines show the "fuzzy" color-magnitude cuts that define the YSO candidate criterion in the various color-magnitude spaces. The red dot-dashed lines show hard limits, fainter than which objects are excluded from the YSO category.

detected or not detected with the same probabilities as those for similar sources in Serpens. The photometric uncertainties of all sources in the resampled catalog of reddened SWIRE sources are reassigned uncertainties similar to those of Serpens sources with similar magnitudes.
Finally, each source in the resampled SWIRE catalog is reclassified, based on its degraded photometry, e.g., "star," "YSOc," "GALc,". . . . The magnitudes, colors, and classifications of sources in this resampled SWIRE catalog are then directly comparable to those in our Serpens catalog and may be used to estimate the 
TABLE 1

YSO versus Extragalactic Discrimination Criteria

\begin{tabular}{|c|c|}
\hline Criterion & Contaminant Probability \\
\hline$K-[4.5]$. & $\operatorname{Prob} /(K-[4.5])$ \\
\hline$[4.5]($ for $[4.5]-[8.0]<0.5) \ldots .$. & Smooth increase for $[4.5]>14.5$ \\
\hline 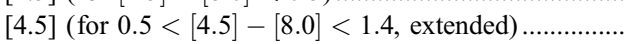 & Smooth increase for $[4.5]>12.5$ \\
\hline$[4.5]$ (for $0.5<[4.5]-[8.0]<1.4$, pointlike). & Smooth increase for $[4.5]>14.5$ \\
\hline 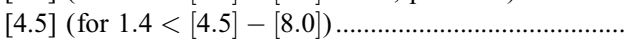 & Smooth increase for $[4.5]>13.0$ \\
\hline$([8.0]-[24]-3.5)^{2} / 1.7+([24]-9.0)^{2} / 2 \ldots \ldots \ldots \ldots \ldots \ldots \ldots$ & Smooth increase for values $<1$ \\
\hline 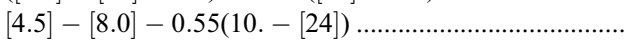 & Smooth increase for values $>1$ \\
\hline [24] (for all colors) & $100 \%$ probability for $[24]>10.0$ \\
\hline Extended at 3.6 or $4.5 \mu \mathrm{m}$ & Twice as likely to be extragalactic \\
\hline$F_{70}$ & Extragalactic prob $\times 0.1$ for $F_{70}>400 \mathrm{mJy}$ \\
\hline
\end{tabular}

population of extragalactic sources satisfying various color and magnitude criteria. At this level of the classification, the terms YSOc and GALc imply candidate classification status.

\subsection{Classification Based on Color and Magnitude}

In Paper II we described a simple set of criteria that basically categorized all objects that were faint and red in several combinations of IRAC and MIPS colors as likely to be galaxies (after removal of normal reddened stars). In our new classification we have extended this concept to include the color and magnitude spaces in Figure 3 together with several additional criteria to compute a proxy for the probability that a source is a $\mathrm{YSO}$ or a background galaxy. Figure 3 shows a collection of three colormagnitude diagrams and one color-color diagram used to classify the sources found in our 3.6-70 $\mu \mathrm{m}$ survey of the Serpens cloud that had $\mathrm{S} / \mathrm{N} \geq 3$ in all the Spitzer bands between 3 and $24 \mu \mathrm{m}$ and that were not classified as reddened stellar photospheres. In addition to the Serpens sources shown in the left panels, the comparable set of sources from the full-sensitivity SWIRE catalog are shown in the middle panels, and the sources remaining in the extincted/sensitivity-resampled version of the SWIRE catalog described above are shown in the right panels. The exact details of our classification scheme are described in the Appendix. Basically we form the product of individual probabilities from each of the three color-magnitude diagrams in Figure 3 and then use additional factors to modify that total "probability" based on source properties such as its $K-[4.5]$ color, whether it was found to be extended in either of the shorter IRAC bands, and whether its flux density is above or below some empirically determined limits in several critical bands. Table 1 summarizes the criteria used for this class separation. The cutoffs in each of the color-magnitude diagrams and the final probability threshold to separate YSOs from extragalactic objects were chosen (1) to provide a nearly complete elimination of all SWIRE objects from the YSO class and (2) to maximize the number of YSOs selected in Serpens consistent with visual inspection of the images to eliminate obvious extragalactic objects (such as a previously uncataloged obvious spiral galaxy at R.A. $=18^{\mathrm{h}} 29^{\mathrm{m}} 57.4^{\mathrm{s}}$, decl. $=$ $+00^{\circ} 31^{\prime} 41^{\prime \prime}$ [J2000.0]).

The cutoffs in color-magnitude space were constructed as smooth, exponentially decaying probabilities around the dashed lines in each of the three diagrams. Sources far below the lines were assigned a high probability of being extragalactic contamination with a smoothly decreasing probability to low levels well above the lines. In the case of the [24] versus [8.0] - [24] relation, the probability dropped off radially away from the center of the elliptical segment shown in the figure. After inclusion of these three color-magnitude criteria, we added the additional criteria listed in Table 1. These included (1) a factor dependent on the $K-[4.5]$ color, prob/ $(K-[4.5])$, to reflect the higher probability of a source being an extragalactic (GALc) contaminant if it is bluer in that color; (2) a higher GALc probability for sources that are extended at either 3.6 or $4.8 \mu \mathrm{m}$, where our survey had the best sensitivity and highest spatial resolution; (3) a decrease in GALc probability for sources with a $70 \mu \mathrm{m}$ flux density above $400 \mathrm{mJy}$, empirically determined from examination of the SWIRE data; and (4) identification as "extragalactic" for any source fainter than [24] =10.0. Again, we emphasize that these criteria are based only on the empirical approach of trying to characterize the SWIRE population in colormagnitude space as precisely as possible, not on any kind of modeling of the energy distributions.

Figure 4 graphically shows the division between YSOs and likely extragalactic contaminants. The number counts versus our probability are shown for both the Serpens cloud and the resampled SWIRE catalog (normalized to the Serpens area). This

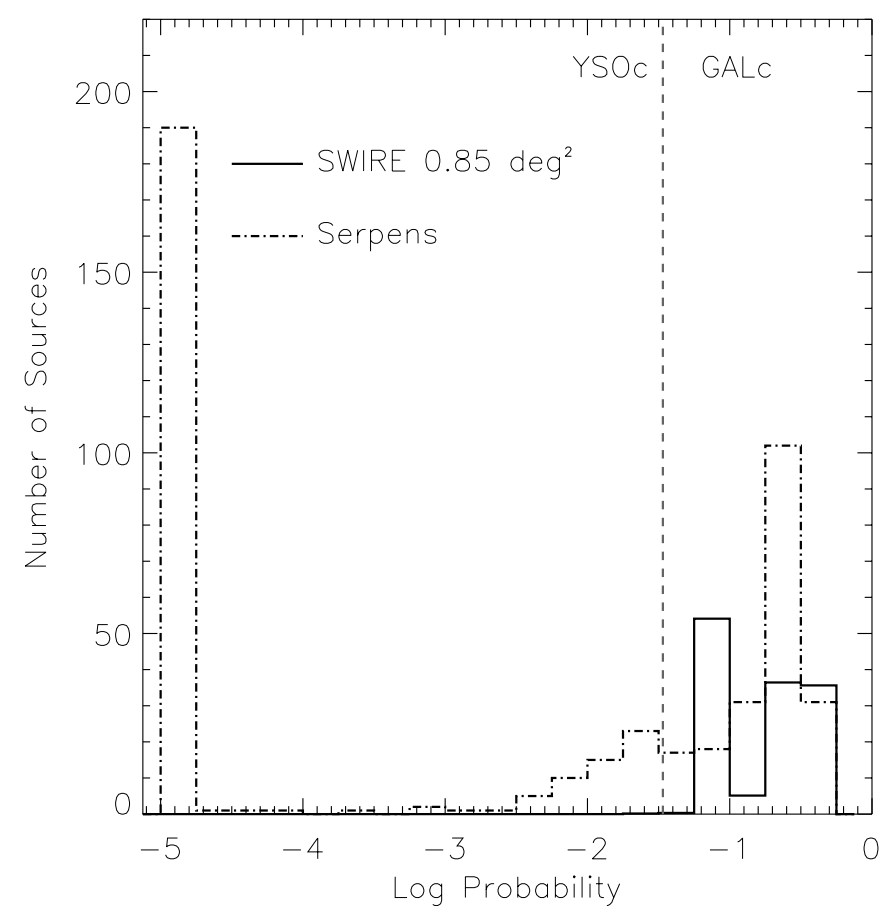

FIG. 4.- Plot of the number of sources vs. probability (of being a background contaminant) for the Serpens cloud and for the trimmed SWIRE catalog described in the text. The vertical dashed line shows the separation chosen for YSOs vs. extragalactic candidates. For both samples, only sources with detections in all four IRAC bands are plotted to keep the number counts of contaminants on scale! 
TABLE 2

YSOS IN SERPENS

\begin{tabular}{|c|c|c|c|c|c|c|c|c|}
\hline ID & Name/Position & Previous Name $^{\mathrm{a}}$ & $\begin{array}{c}3.6 \mu \mathrm{m} \\
(\mathrm{mJy})\end{array}$ & $\begin{array}{c}4.5 \mu \mathrm{m} \\
(\mathrm{mJy})\end{array}$ & $\begin{array}{c}5.8 \mu \mathrm{m} \\
(\mathrm{mJy})\end{array}$ & $\begin{array}{c}8.0 \mu \mathrm{m} \\
(\mathrm{mJy})\end{array}$ & $\begin{array}{c}24.0 \mu \mathrm{m} \\
(\mathrm{mJy})\end{array}$ & $\begin{array}{c}70.0 \mu \mathrm{m} \\
(\mathrm{mJy})\end{array}$ \\
\hline & SSTc2d J18275381-0002333 & $\ldots$ & $25.5 \pm 1.2$ & $20.2 \pm 1.0$ & $15.6 \pm 0.7$ & $13.9 \pm 0.7$ & $22.7 \pm 2.1$ & \\
\hline $3 \ldots \ldots \ldots \ldots$ & SSTc2d J18280845-0001064 & $\ldots$ & $140 \pm 7$ & $108 \pm 8$ & $129 \pm 6$ & $122 \pm 8$ & $161 \pm 14$ & $314 \pm 36$ \\
\hline $4^{\mathrm{b}} \ldots \ldots \ldots$ & SSTc2d J18281100-0001393 & $\ldots$ & $104 \pm 5$ & $63.4 \pm 3.2$ & $54.6 \pm 2.6$ & $35.4 \pm 1.9$ & $9.51 \pm 0.90$ & $\ldots$ \\
\hline $5 \ldots \ldots \ldots \ldots$ & SSTc2d J18281350-0002491 & CDF 88-2 & $105 \pm 5$ & $88.1 \pm 4.7$ & $77.5 \pm 3.7$ & $94.3 \pm 5.0$ & $254 \pm 23$ & $382 \pm 43$ \\
\hline $8 \ldots \ldots \ldots \ldots .$. & SSTc2d J18281525-0002432 & CoKu Ser-G1 & $174 \pm 9$ & $177 \pm 9$ & $180 \pm 11$ & $191 \pm 11$ & $1200 \pm 25$ & $1670 \pm 169$ \\
\hline $9 \ldots \ldots \ldots . .$. & SSTc2d J18281628-0003161 & $\ldots$ & $53.1 \pm 2.7$ & $48.4 \pm 2.4$ & $45.4 \pm 2.1$ & $46.2 \pm 2.5$ & $74.7 \pm 6.9$ & $129 \pm 24$ \\
\hline $10 \ldots \ldots \ldots$ & SSTc2d J18281852-0003329 & $\ldots$ & $3.38 \pm 0.17$ & $3.14 \pm 0.15$ & $3.13 \pm 0.16$ & $2.99 \pm 0.15$ & $2.96 \pm 0.36$ & $\ldots$ \\
\hline
\end{tabular}

Notes.-Table 2 is published in its entirety in the electronic edition of the Astrophysical Journal. A portion is shown here for guidance regarding its form and content. a Source names from SIMBAD, including numbers from the following catalogs: EC: Eiroa \& Casali (1992); D: Djupvik et al. (2006); K: Kaas et al. (2004).

${ }^{\mathrm{b}}$ May be an AGB star, based on $A_{v}$ derived from optical spectrum.

illustrates how cleanly the objects in the SWIRE catalog are identified by this probability criterion. In the Serpens sample there are clearly two well-separated groups of objects plus a tail of intermediate-probability objects that we have mostly classified as YSOs since no such tail is apparent in the SWIRE sample.

Our choice of the exact cut between "YSO" and "XGal" in Figure 4 is somewhat arbitrary because of the low-level tail of objects in Serpens in the area of $\log$ (probability) $\sim-1.5$. Since the area of sky included in our SWIRE sample is more than 6 times as large as the mapped area of Serpens, we chose our final probability cut, $\log P \leq-1.47$, to allow two objects from the full (not resampled) SWIRE catalog into the "YSO" classification bin. Thus, aside from the vagaries of small number statistics, we expect of order 0-1 extragalactic interlopers in our list of Serpens YSOs. The right panels of Figure 3, which use the resampled version of the SWIRE catalog, show that the effects of sensitivity and, especially, the extinction in Serpens make our cutoff limits particularly conservative in terms of likelihood of misclassification. We examined all the YSO candidates chosen with these criteria in terms of both the quality of the photometry and their appearance in the images. A significant number, $\sim 50$ candidates, were discarded because of the poor quality of the band-filling process at $24 \mu \mathrm{m}$ due to contamination by a nearby brighter source or because of their appearance in the images. It is possible that a small number of these discarded candidates are, in fact, true YSOs in the Serpens cloud. We also manually classified one source as "YSO" (number 75 in Table 2) that may be the exciting source for an HH-like outflow in cluster B (see also discussion of this region by Harvey et al. 2007), but which was not so classified because of its extended structure in the IRAC bands. Table 2 lists the 235 YSOs that resulted from this selection process.

Another test of the success of our separation of background contaminants is to simply plot the locations of the YSOs and likely galaxies on the sky. Figure 5 shows such a plot in addition to a plot of visual extinction discussed later. There is clearly a very uniform distribution of background contaminants and a quite clustered distribution of YSOs (see further discussion in $\S 7)$.

There is one additional kind of contaminant that is likely to appear in our data at a relatively low level, asymptotic giant branch (AGB) stars. The ISO observations by, for example, van Loon et al. (1999) and Trams et al. (1999) show that the range of brightnesses of AGB stars in the LMC covers a span equivalent to roughly $8<[8.0]<12$, with colors generally equivalent to
$[4.5]-[8.0]<1$. Very compact proto-planetary nebulae are generally redder, but even brighter at $8 \mu \mathrm{m}$ (see e.g., Hora et al. 1996). For typical Galactic AGB stars, between 5 and $15 \mathrm{kpc}$ from the Sun, this would imply $3<[8.0]<9$. The off-cloud fields (when normalized to the same area as the Serpens data) provide the best handle on the degree of contamination from AGB stars; in Paper II we saw one object classified as a YSO candidate in the off-cloud panel of Figure 9 below with $[8.0]<9$. Therefore, based on the ratio of areas mapped in Serpens versus the off-cloud observations, we expect the number of AGB stars contaminating the YSO candidate list in the Serpens cloud to be of order a halfdozen. As a further check, we have examined the entire set of offcloud fields for such objects. In this combined area of $0.58 \mathrm{deg}^{2}$, there are only three such YSO candidates. Finally, B. Merín et al. (2007, in preparation) have classified four AGB stars by their Spitzer IRS spectra in the Serpens cloud; these are identified in the diagrams of Figure 3 by black open diamonds and obviously are not included in our final list of high-probability YSOs. Statistically then, it is possible that a couple of our brightest YSOs are in fact AGB stars. In fact, B. Merín et al. (2007, in preparation) have obtained modest $\mathrm{S} / \mathrm{N}$ optical spectra of a number of our YSOs. They find five objects whose estimated extinction seems inconsistent with their location in the Serpens cloud and that suggests that they might be background objects at much larger distances with modest circumstellar shells like AGB stars. We have indicated these five objects in Table 2 with footnote $b$.

It is also interesting to ask the reverse question, to what extent have our criteria successfully selected objects that were known YSOs from previous observations. Alcalá et al. (2007) have used the same criteria to search for YSOs in the c2d data for Chamaeleon II. They conclude that all but one of the known YSOs in that region are identified. In $\S 5$ we discuss the comparison of our results with several previous studies of Serpens that searched for YSOs. Our survey found counterparts to all the previously known objects in our observed area that had $\mathrm{S} / \mathrm{N}>5$ in those studies, but we did not classify many of them as YSOs because of the lack of significant excess in the IRAC or MIPS bands. Most of these were indeed suggested only as candidate YSOs by the authors, so we do not consider this fact to be a problem for our selection criteria. We again emphasize the fact that our selection criteria for youth are based solely on the presence of an infrared excess at some Spitzer wavelength.

The total number of objects classified as YSOs in the IRAC/ MIPS overlap area of the Serpens cloud is 235. From the statistics of our classification of the SWIRE data we expect that of 


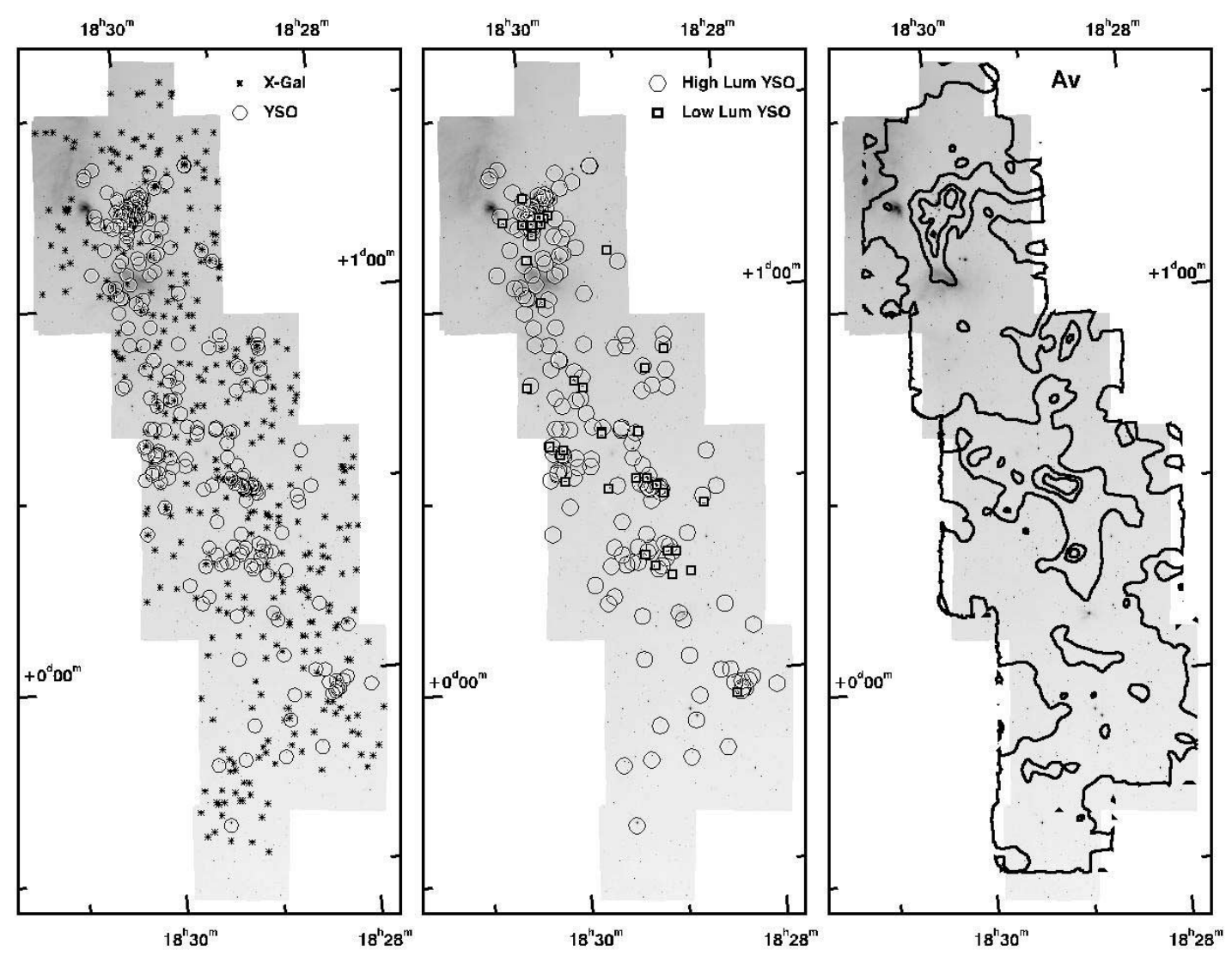

FIG. 5. - Left: Image of the entire mapped area of Serpens at $8.0 \mu \mathrm{m}$ with the positions of YSOs plotted with circles and likely extragalactic background contaminants with asterisks. Middle: Same image with YSOs of luminosity $L<2 \times 10^{-2} L_{\odot}$ plotted with squares and higher luminosity YSOs plotted with circles. Right: Contours of visual extinction $A_{v}$ at levels of 5,10,20, and $30 \mathrm{mag}$ as derived from fitting the energy distributions of sources that were well fitted as reddened stellar photospheres in our data set.

order $1 \pm 1$ of these are likely to be galaxies. On the other hand, as we discuss in $\S 6$, some of the faint red objects in Figure 3 (below the dot-dashed line) may also be young substellar objects. For example, we note the case of the young brown dwarf BD-Ser 1 found by Lodieu et al. (2002) that was not selected by our criteria because of its relative faintness. Of these 235, 198 were detected in at least the $H$ and $K_{s}$ bands of 2MASS at better than $7 \sigma$. The number of YSOs in each of the four classes of the system suggested by Lada (1987) and extended by Greene et al. (1994) is 39 Class I, 25 "flat," 132 Class II, and 39 Class III, using the flux densities available between 1 and $24 \mu \mathrm{m}$. Because we require some infrared excess to be identified as a YSO, the Class III candidates were necessarily selected by some measurable excess typically at the longer wavelengths, 8 or $24 \mu \mathrm{m}$. An obvious corollary is that objects identified as young on the basis of other indicators (e.g., X-ray emission, lithium abundance) but without excesses in the range of 1-24 $\mu \mathrm{m}$ are not selected with our criteria.

\subsection{YSOs Selected by MIPS}

Harvey et al. (2007) found 250 YSO candidates in the entire area mapped by MIPS at $24 \mu \mathrm{m}$ in Serpens, an area of $1.8 \mathrm{deg}^{2}$; 51 of these are outside the IRAC/MIPS overlap area and are listed in Table 3. We can make a comparison of those statistics with the YSO counts here in two ways. First, in the area covered by both IRAC and MIPS-24 there are 197 objects that satisfy the criteria of Harvey et al. (2007), i.e., $K_{s}<14, K_{s}-[24]>2$, $[24]<10$, and $24 \mu \mathrm{m} \mathrm{S} / \mathrm{N} \geq 5$. Of these, 184 satisfy our more restrictive criteria in this study based on the combination of 2MASS, IRAC, and MIPS data, or $93 \%$. We would classify the other 13 as likely background galaxies. Secondly, of the 235 YSOs found in this study, 200 have sufficient data to be classifiable by the "MIPS-only" criteria above, but only 167 or $84 \%$ actually meet the MIPS-only YSO criteria. In other words, $33 \mathrm{ob}-$ jects have been classified as high-quality YSOs in this paper in the MIPS/IRAC overlap region that did not meet the criteria based only on MIPS and 2MASS data. If these ratios can be extrapolated to the larger area covered only by MIPS, then we would expect Harvey et al. (2007) to have missed 16\% (eight or nine) of the YSOs but to have included 7\% (three or four) that would not meet our combined IRAC/MIPS criteria. With these corrections we might have expected to find $235+56=291$ YSOs in the entire $1.8 \mathrm{deg}^{2}$ area covered by MIPS if we had matching IRAC observations. Finally, in light of our earlier discussion of AGB contaminants, it is possible that six to nine of these YSOs would actually be found to be background AGB stars.

\section{SEARCH FOR VARIABILITY}

The fact that our data were taken in two epochs separated by $6 \mathrm{hr}$ or more gives us the opportunity to search for variability over that timescale. Rebull et al. (2007) and Harvey et al. (2007) have performed similar tests for variability of the $24 \mu \mathrm{m}$ emission from sources in the c2d observations of Perseus and Serpens and found no reliable evidence for variability at that wavelength. There is, however, substantial evidence for short-term variability in the near-infrared for YSOs. We therefore performed a similar 
TABLE 3

YSO Candidates in Serpens without Four-Band IRAC Observations

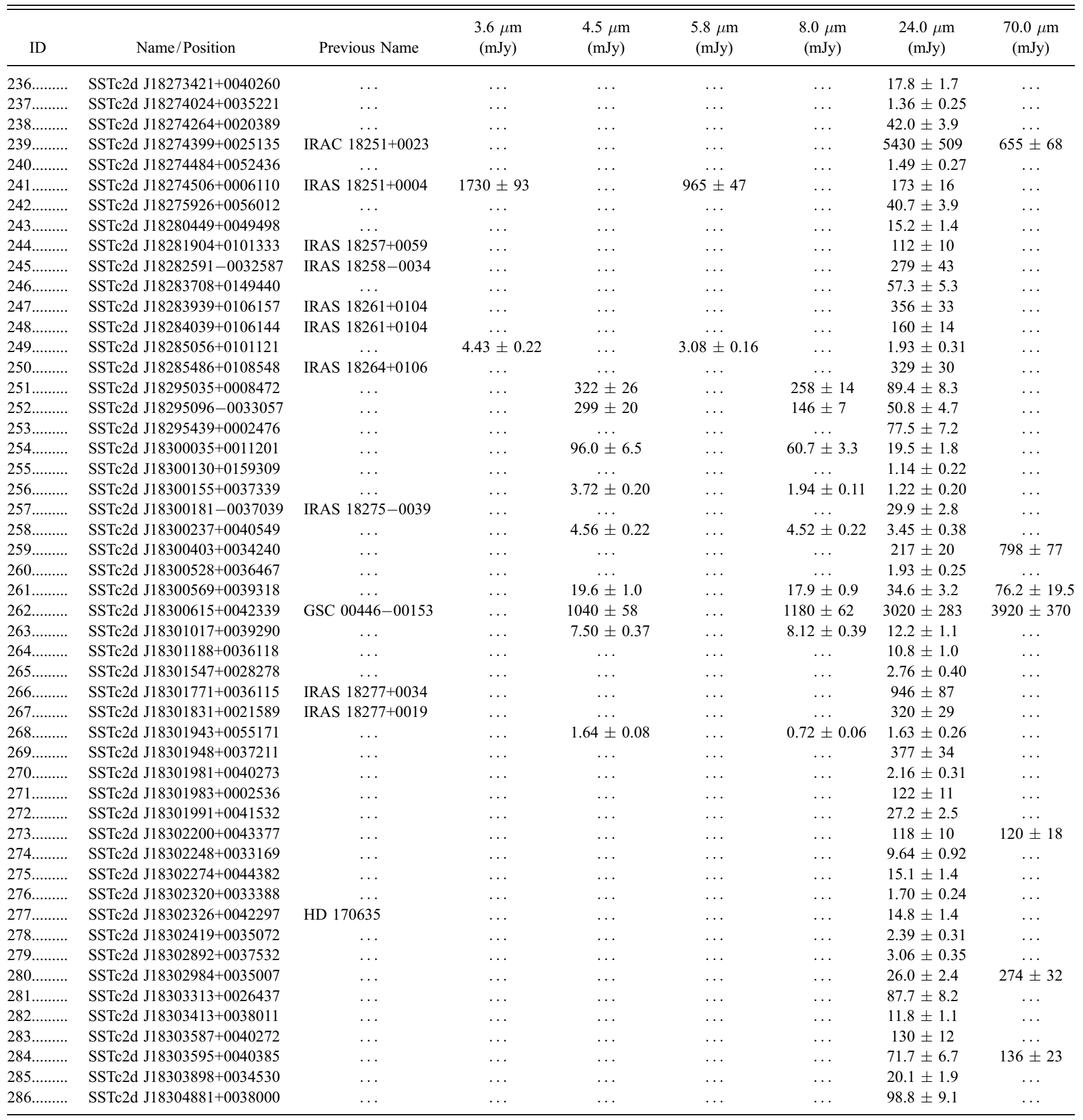

investigation in the two shortest IRAC bands, 3.6 and $4.5 \mu \mathrm{m}$. No clear evidence was found at the level of $\pm 25 \%$ for any sources in the field over the $6 \mathrm{hr}$ timescale of our multiepoch observations.

\section{COMPARISON WITH PREVIOUS STUDIES OF SERPENS}

We have cross-correlated our source catalog with those from previous studies of Serpens that searched for YSOs. We chose three studies that covered much of cluster A at near-IR, ISO, and
X-ray wavelengths (Eiroa \& Casali 1992; Kaas et al. 2004; Preibisch 2003) and one recent $I S O$ study of cluster B (Djupvik et al. 2006). Table 4 lists the sources from each of these previous studies and the best-matching Spitzer source from our complete catalog. In brief, we find good matches for essentially all the previous IR-selected YSOs that had $\mathrm{S} / \mathrm{N}>4$ in the earlier studies and that were included in our mapped area. In detail, however, a number of YSO candidates from the earlier studies were not classified as YSOs in our study. The reasons for this are different for the various catalogs. From the X-ray catalog of Preibisch 
TABLE 4

Matching Spitzer Sources/Fluxes for Previously Identified IR Sources in Serpens

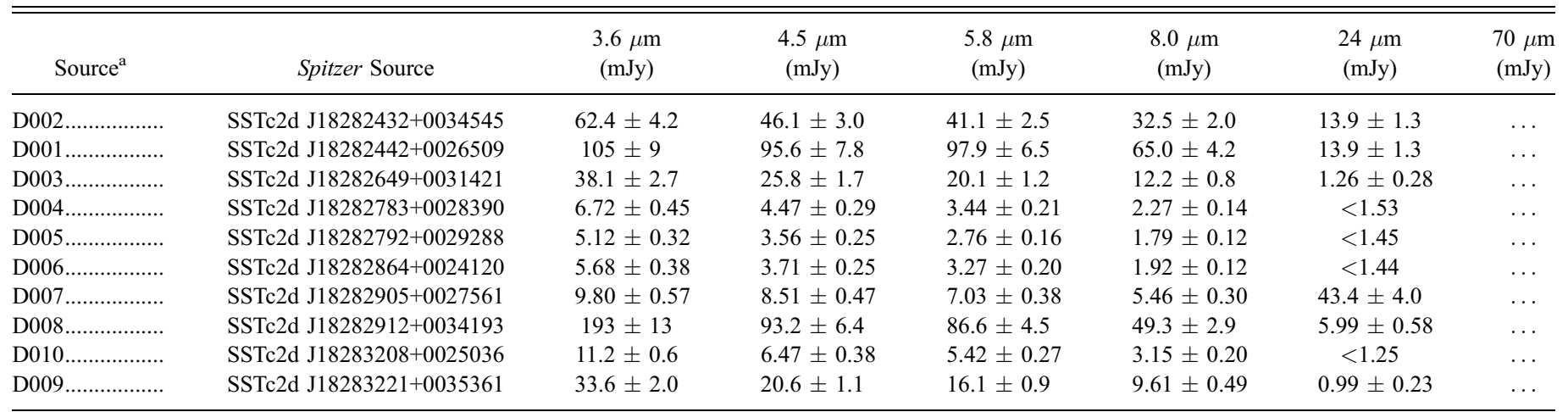

Notes.- - Table 4 is published in its entirety in the electronic edition of the Astrophysical Journal. A portion is shown here for guidance regarding its form and content.

${ }^{a}$ Source numbers from the following catalogs: EC: Eiroa \& Casali (1992); D: Djupvik et al. (2006); P: Preibisch (2003); K: Kaas et al. (2004).

(2003) in cluster A, we only identified 17 of the 45 X-ray sources as YSOs on the basis of their infrared excesses. The X-ray sources that were not identified as YSOs included both many objects that were well fitted as reddened stellar photospheres (21 sources) and objects with some likely infrared excess but too little to fit our criteria aimed at eliminating extragalactic interlopers. The situation with the infrared catalogs is somewhat different. Examining our nonmatches from the ground-based study of Eiroa \& Casali (1992), we find that a large fraction of their YSOs are classified as such by our criteria, but not all. From the ISO surveys (Kaas et al. 2004; Djupvik et al. 2006) we typically identify $\sim 50 \%-60 \%$ as YSOs. The ones that we do not classify as such are typically those with low $\mathrm{S} / \mathrm{N}$ in the $I S O$ observations or where the amount of infrared excess was not large enough to satisfy our test of whether the object could not be fitted as an extincted stellar photosphere as described by Evans et al. (2007).

\section{LUMINOSITIES}

\subsection{The YSO Luminosity Function in Serpens}

One of the most important physical parameters for any star is its mass. For pre-main-sequence stars this is problematic because the determination of mass depends on the placement of the star on an H-R diagram and the use of model evolutionary tracks for which there is significant uncertainty at the level of at least a factor of 1.5-2 in the literature (Hillenbrand \& White 2004; Stassun et al. 2004). For young stars in a cluster, a poor but still useful proxy for mass is the stellar luminosity since it is possible that most of the stars have formed more or less simultaneously. With all these caveats in mind, we display in Figure 6 the histogram of total luminosities for the 235 YSOs found in Serpens in our survey with the assumed distance of $260 \mathrm{pc}$. We remind the reader that these objects were selected specifically on the basis of infrared excess emission, so this list is limited to YSOs that show substantial IR excess at least somewhere in the range of 3.5-70 $\mu \mathrm{m}$. For example, as we discussed in $\S 5$, a comparison of our list of YSOs with the list of X-ray sources in cluster A (Preibisch 2003) showed that more than half the X-ray sources were not classified as YSOs by our infrared excess criteria. In some sense then, it is likely that the IR-selected and X-rayselected samples are complementary in selecting less evolved and more evolved samples of YSOs, respectively. The luminosity function of our IR excess-defined YSO sample peaks at roughly $2 \times 10^{-2} L_{\odot}$ and drops off steeply below $10^{-2} L_{\odot}$. This is interestingly equal to the luminosity of objects at the hydrogenburning limit of $0.075 M_{\odot}$ in the models of Baraffe et al. (2002) for objects with ages of $\sim 2 \mathrm{Myr}$, a common estimate for the age of the Serpens star-forming event (Kaas et al. 2004; Djupvik et al. 2006). Of course, an important question to ask is to what extent this luminosity distribution in Figure 6 is influenced by selection effects.

We can estimate the selection effects in our sample from the statistics in the SWIRE samples discussed above. The completeness limits of the full catalog are nearly 2 mag fainter than the trimmed version, so we assume for simplicity that the full catalog is $100 \%$ complete down to the faintest magnitudes of interest for this test in the $\mathrm{c} 2 \mathrm{~d}$ Serpens data set. Figure 7 shows the number counts as a function of "luminosity" (all sources assumed to be at $260 \mathrm{pc}$ ) for three versions of our c2d-processed SWIRE catalog: (1) the full-depth catalog, (2) the catalog cut off with c2d sensitivity limits but without added extinction, and (3) the catalog corrected for both c2d sensitivity levels and extinction in the Serpens cloud. The ratio of the second to the first version serves as a good proxy for a completeness function since it includes the

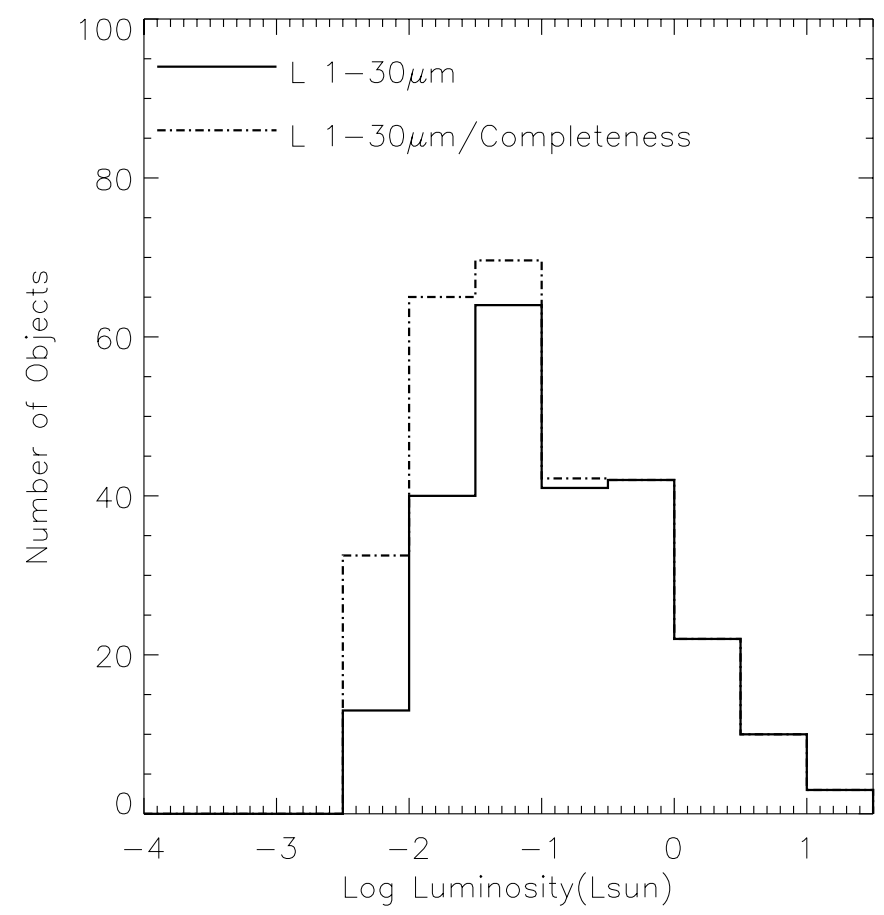

FIG. 6.- Luminosity function for Serpens YSOs (solid histogram) and estimate of correction for completeness effects (dot-dashed histogram). 


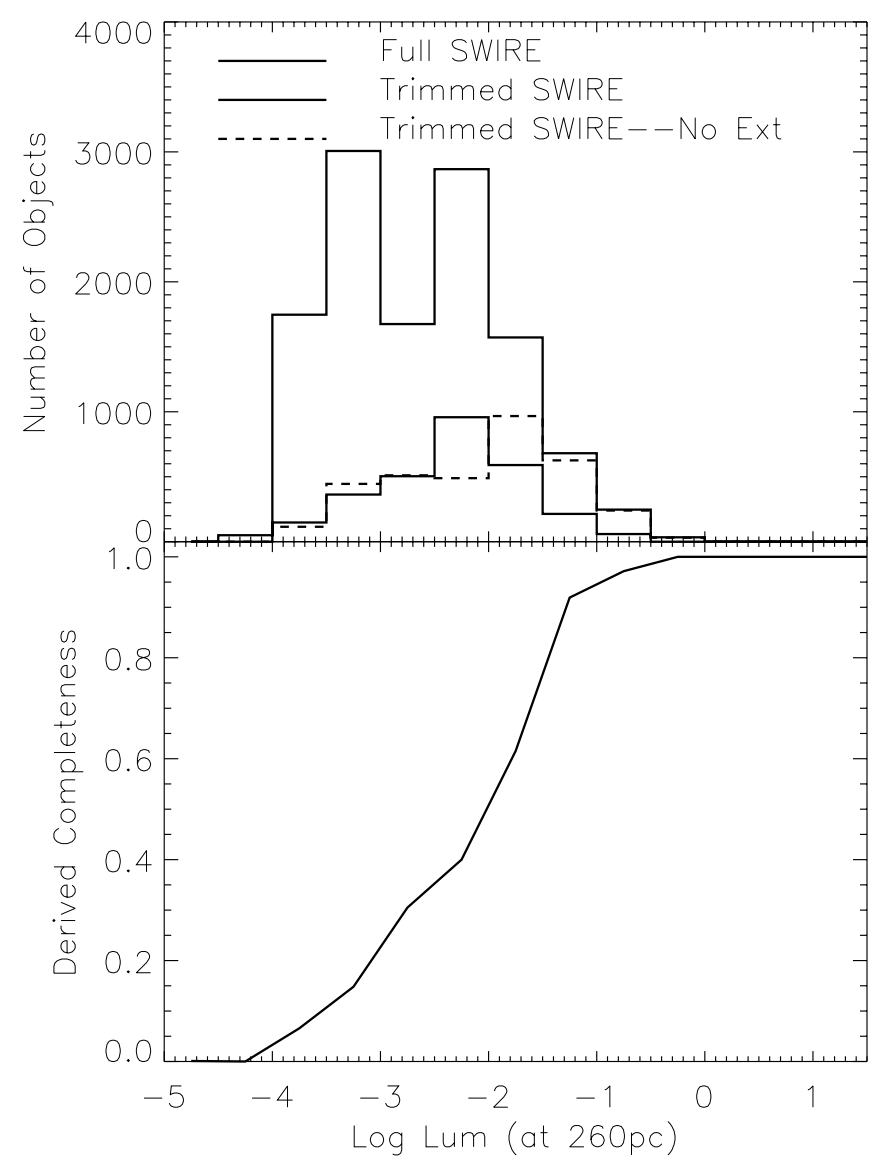

FIG. 7.-Top: Number counts for the full SWIRE catalog as processed through the $\mathrm{c} 2 \mathrm{~d}$ pipeline for objects detected in all four IRAC bands vs. those for the "trimmed" version of the catalog with completeness limits for each individual band comparable to those for $\mathrm{c} 2 \mathrm{~d}$. Bottom: Ratio of the two number counts, i.e., completeness factor.

effects of $\mathrm{c} 2 \mathrm{~d}$ sensitivity only. The bottom panel of Figure 7 then shows this ratio as our estimate of the completeness function at the range of luminosities of Serpens YSOs observed in Figure 6. In Figure 6, then, we also indicate how the YSO luminosity function might be adjusted to account for this estimate of our survey completeness (with an assumed completeness of $100 \%$ at the bright end). Although substantial adjustments must be made to the number counts at fainter luminosities, the general conclusion remains intact that the luminosity function peaks around a few times $10^{-2} L_{\odot}$ and drops to both lower and higher luminosities. Interestingly, if our completeness estimates are valid, there may still be a substantial population of IR excess sources down to luminosities of $10^{-3} L_{\odot}$ as was already suggested in Paper II. For example, as noted earlier in $\S 3.2$, the young brown dwarf found by Lodieu et al. (2002) was not selected as a YSO with our criteria because it was faint enough that its colors placed it into the color and magnitude ranges where extragalactic objects begin to be prominent.

\subsection{The Lowest Luminosity Sources}

There are 37 YSOs with total luminosities $(1-70 \mu \mathrm{m})$ less than $2 \times 10^{-2} L_{\odot}$, without any correction for selection effects in either our observations or our selection criteria. Figure 5 presented in $\S 3.2$ shows the spatial distribution of the low- $(L<2 \times$ $\left.10^{-2} L_{\odot}\right)$ and "high"-luminosity $\left(L>2 \times 10^{-2} L_{\odot}\right)$ YSOs in Serpens. There does not appear to be any significant difference between these two distributions. Likewise, Figure 8 shows the

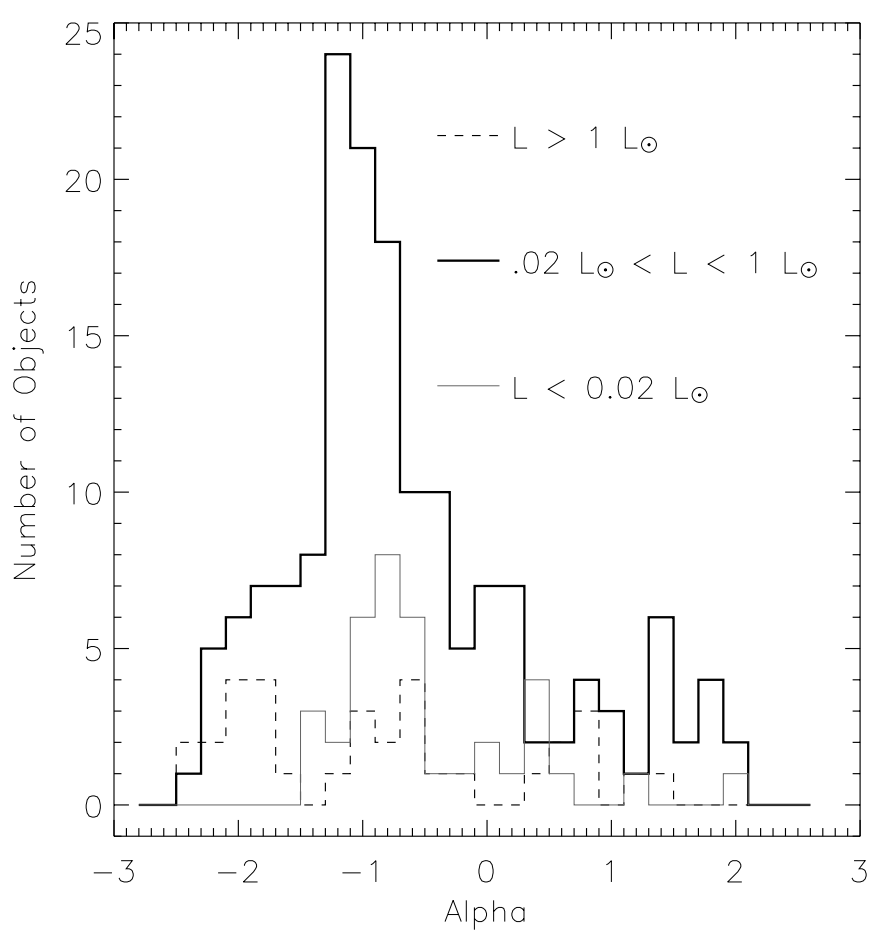

FIG. 8.- Histogram of distribution of spectral slopes, "alpha," for three luminosity "classes" of YSOs.

distribution of spectral slopes, $\alpha$, for the low-luminosity sample relative to two higher luminosity samples, and Table 5 lists the average and standard deviation for the spectral slopes for three luminosity samples. Again, there is no obvious difference in the distributions within the statistical uncertainties of the samples (see also discussion in $\S 8.1$ ). These two facts suggest that the mechanisms and timing of formation of these two luminosity groups may not be very different. Finally, Figure 9 shows the distribution of extinction for stars nearby each YSO. For this analysis we selected all objects within $80^{\prime \prime}$ of each YSO that were classified as "star" by the process described earlier. We averaged the fitted extinction values for these stars; the number of stars contributing to the average ranged from 5 to 48 . This figure shows that the lower luminosity YSOs have at least as much typical extinction as the higher luminosity ones, a fact that would be unlikely if they were misclassified background extragalactic objects.

\section{SPATIAL DISTRIBUTION OF STAR FORMATION}

We have already noted in $\S 1$ that the Serpens star-forming region has been identified as one displaying strong evidence of clustering of the youngest objects. We also showed in Figure 5 that the spatial distribution of our high-quality YSO candidates was highly clustered. A number of authors have attempted to quantify the degree of clustering as a function of spatial scale using the two-point correlation function (Johnstone et al. 2000; Enoch

TABLE 5

Average/Standard Deviation of Spectral Slope $\alpha$ VERSUS LUMINOSITY OF YSOS

\begin{tabular}{|c|c|c|}
\hline Luminosity Range & Average $\alpha$ & $\sigma_{\alpha}$ \\
\hline$<0.02 L_{\odot} \ldots \ldots \ldots \ldots \ldots \ldots \ldots \ldots$ & -0.73 & 1.47 \\
\hline $0.02-1.0 L_{\odot} \ldots \ldots \ldots \ldots \ldots \ldots \ldots$ & -0.70 & 1.04 \\
\hline$>1.0 L_{\odot} \ldots \ldots \ldots \ldots \ldots \ldots \ldots \ldots \ldots$ & -0.60 & 0.74 \\
\hline
\end{tabular}




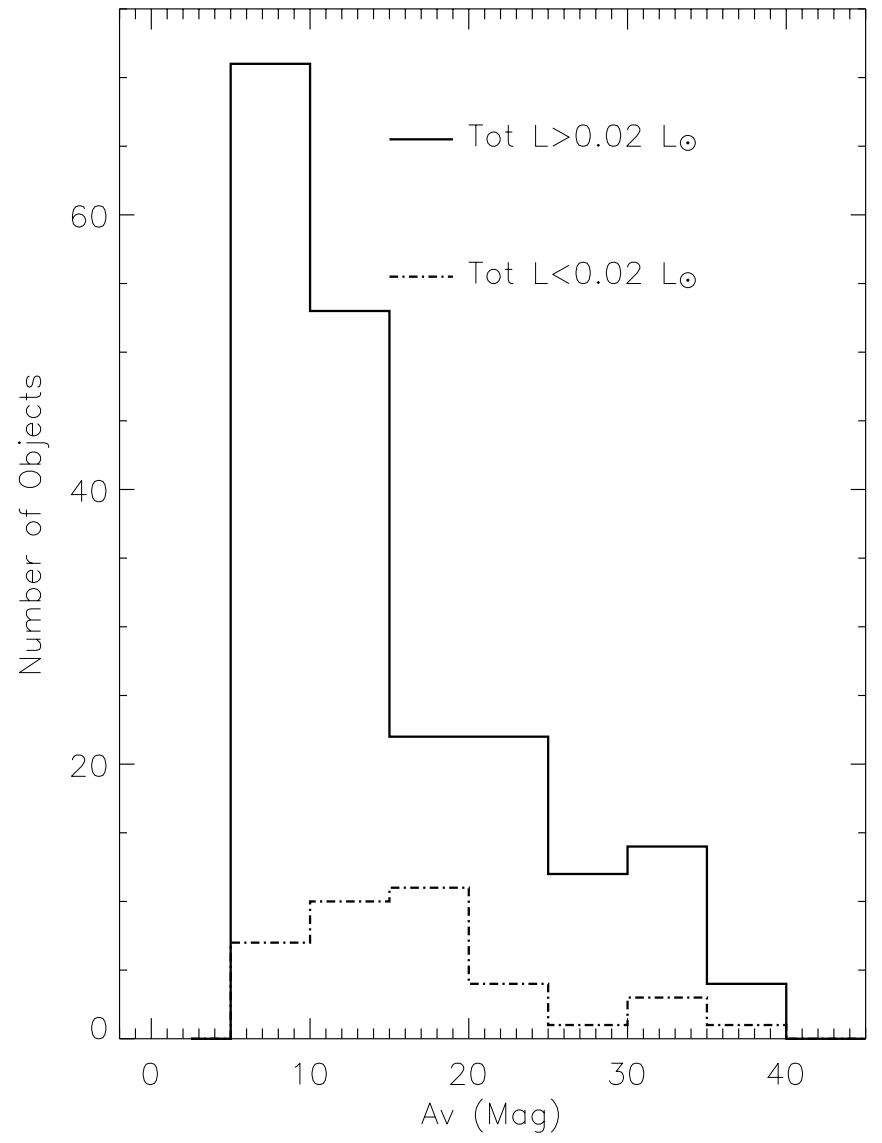

FIG. 9.- Histogram of distribution of average extinction for stars within $80^{\prime \prime}$ of each YSO for "high"- and "low"-luminosity YSOs (above or below $\left.2 \times 10^{-2} L_{\odot}\right)$. et al. 2006a; Simon 1997; Gomez \& Lada 1998; Bate et al. 1998). Typical results have found a steep slope, $\gamma \propto d^{-2}$, on small spatial scales, implying a rapid decrease in clustering on scales out to $\sim 10,000 \mathrm{AU}$, and a substantially shallower slope, $\gamma \propto d^{-0.5}$, beyond that (Simon 1997; Gomez \& Lada 1998). Figure 10 shows the two-point correlation function, $W$, for the YSOs in our Serpens catalog and the sample of objects classified as extragalactic background sources. The Serpens samples are divided into two groups: those with spectral slopes, $\alpha$, that place them in the Class I or "flat" categories of Greene et al. (1994), and those whose slopes imply Class II or III. Although our range of good sampling only extends down to $\sim 1000 \mathrm{AU}$, equivalent to $4^{\prime \prime}$, our data suggest that the slope for separations under $10^{4} \mathrm{AU}$ is of order 0.5 and drops steeply for the more embedded and likely youngest Class I and flat spectral energy distribution (SED) objects beyond $10^{4}$ AU. The nominally more evolved objects in Classes II and III exhibit a lower level of clustering and more uniform slope in the two-point correlation function. These results are consistent with the appearance of the source distributions as shown, for example, in Figure 13 of Paper II. As shown in Figure 10, the slope and magnitude of the correlation for our sample of background extragalactic objects are consistent with those found by, for example, Maddox et al. (1990) for a bright sample of galaxies.

The total surface density of YSOs in a cluster is an indicator of the richness of star formation in the cluster. Allen et al. (2007) have computed surface densities for YSOs identified by infrared excess in a sample of 10 young clusters. They find typical peak surface densities of 500-1000 $\mathrm{pc}^{-2}$ and average values of order $100-200 \mathrm{pc}^{-2}$. The peak surface density in cluster A of our YSOs identified by IR excess is comparable to these, of order $10^{3} \mathrm{pc}^{-2}$, and a factor of about 2 less in cluster $\mathrm{B}$. The average values are about a factor of 4 less than the peak in both clusters. In particular, if we define the cluster edges by the $A_{v}=20$ contour for comparison with other c $2 \mathrm{~d}$ clusters, we obtain the values

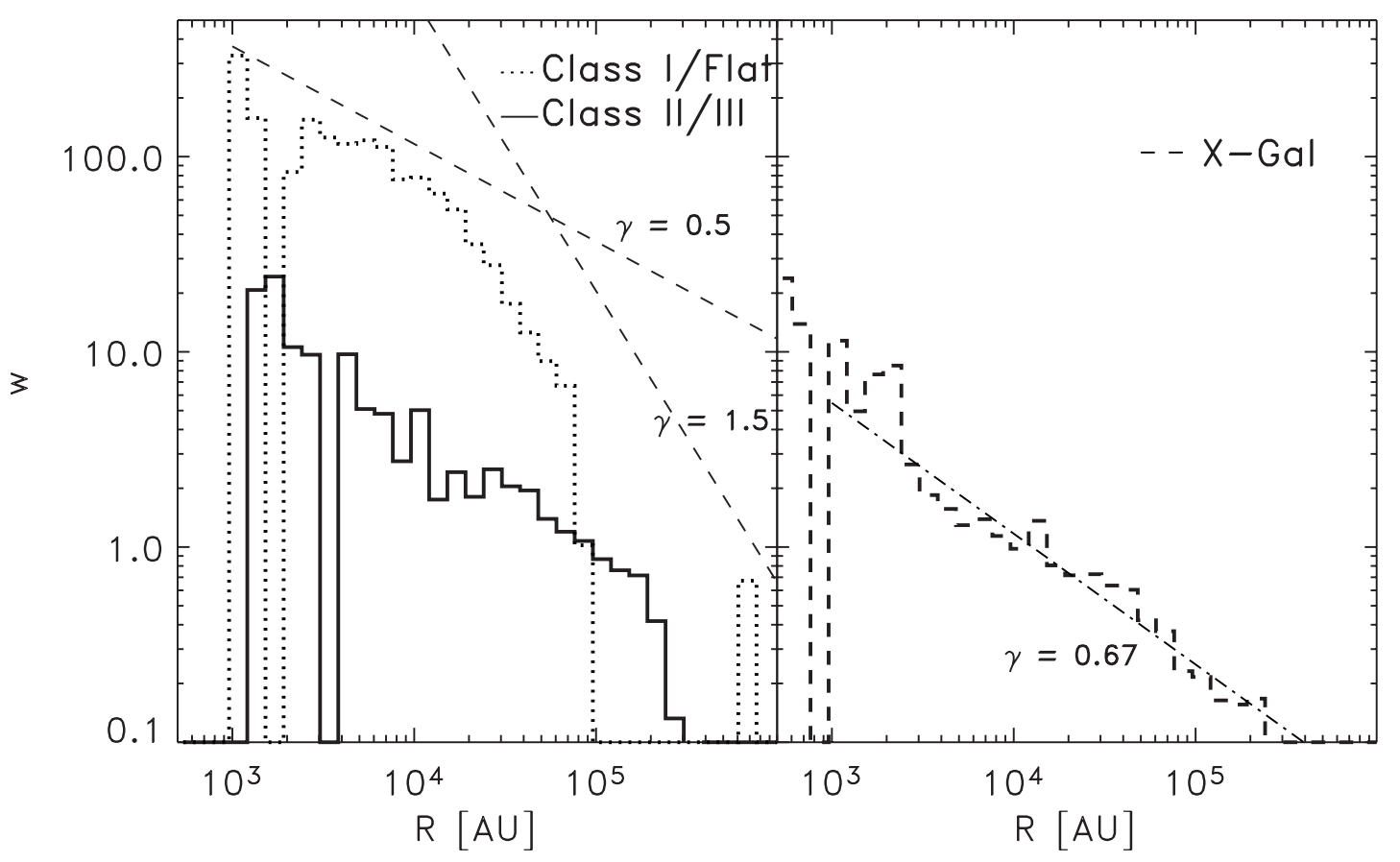

FIG. 10.-Left: Two-point correlation function, $W$, for Serpens. The dotted histogram is for sources identified as Class I and flat SED objects, i.e., $\alpha \geq-0.3$. The

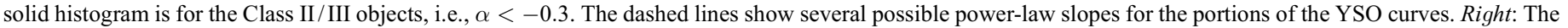

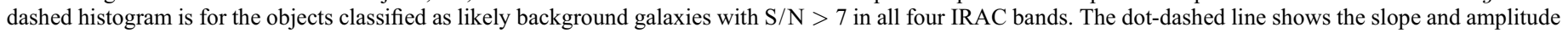

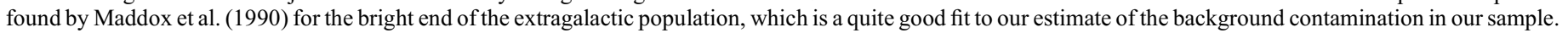


TABLE 6

Numbers, Densities, Star Formation Rates

\begin{tabular}{lccccccc}
\hline \hline Region & Area & $\begin{array}{c}N(\text { YSOs }) \\
\left(\mathrm{pc}^{2}\right)\end{array}$ & $\begin{array}{c}N / \Omega \\
\left(\mathrm{deg}^{-2}\right)\end{array}$ & $\begin{array}{c}N / \text { Area } \\
\left(\mathrm{pc}^{-2}\right)\end{array}$ & $\begin{array}{c}N / \text { Volume } \\
\left(\mathrm{pc}^{-3}\right)\end{array}$ & $\begin{array}{c}\text { SFR } \\
\left(M_{\odot} \mathrm{Myr}^{-1}\right)\end{array}$ & $\begin{array}{c}\text { SFR/Area } \\
\left(M_{\odot} \mathrm{Myr}^{-1} \mathrm{pc}^{-2}\right)\end{array}$ \\
\hline Cluster A .................. & 0.20 & 44 & 4580 & 222 & 500 & 11 & 56 \\
Cluster B ................. & 0.14 & 17 & 2450 & 119 & 315 & 4.3 & 30 \\
Rest of cloud............. & 17.2 & 174 & 209 & 10.1 & 2.5 & 44 & 2.5 \\
All of cloud.............. & 17.5 & 235 & 276 & 13.4 & 3.2 & 59 & 3.4 \\
\hline
\end{tabular}

in Table 6 for the number of stars per solid angle and per square parsec. We have compared the values for clusters A and B with those for the rest of the cloud and for the total cloud surveyed. The surface densities of YSOs are 10-20 times higher in the clusters than in the rest of the cloud. As shown by Allen et al. (2007), cluster A in Serpens is particularly striking in terms of the contrast between the peak surface density and how quickly, within $1 \mathrm{pc}$, the density drops to very low values, around a few $\mathrm{pc}^{-2}$. In terms of volume density, however, even cluster A has a substantially lower volume density of star formation than such rich clusters as the Trapezium $\left(5000 \mathrm{pc}^{-3}\right)$ or Mon R2 $\left(9000 \mathrm{pc}^{-3}\right.$; Elmegreen et al. 2000).

Figure 5 also shows the spatial distribution of visual extinction in the cloud as derived from our data. These extinction values were derived from the combination of 2MASS and Spitzer data for sources that were well fitted as reddened stellar photospheres as described in detail by Evans et al. (2007). As already noted in our previous studies of the IRAC data alone (Harvey et al. 2006) and the MIPS data alone (Harvey et al. 2007), there is clearly a striking correspondence between the areas of high extinction and the densest clusters of YSOs, particularly those containing Class I and flat sources.

\section{DISK PROPERTIES}

The properties of the circumstellar dust surrounding young stars can be estimated from the overall energy distributions of the objects in a variety of ways. These properties include the overall amount of dust, the density distribution as a function of radial distance from the central star, the morphology of the circumstellar disks that probably represent the configuration of many of these circumstellar distributions, and, to some extent, the degree of evolution of the dust from typical interstellar dust to larger and more chemically evolved grains likely to represent some stage in the formation of planetary systems. We discuss several approaches to classifying the dust emission in this section, beginning with the use of infrared colors.

\subsection{Color-Color Diagrams}

Numerous authors have shown how combinations of colors in the infrared can provide significant diagnostic information on the configuration and total amount of circumstellar material around young stars. Whitney et al. (2003) modeled a wide range of disk and envelope configurations and computed their appearance in a number of infrared color-color and color-magnitude diagrams particularly relevant to Spitzer's wavelength range. Similarly, Allen et al. (2004) have published IRAC color-color diagrams for another set of models of objects with circumstellar disks and/or envelopes. Recently, Robitaille et al. (2006) have computed a very large grid of models to extend the predictions from those of Whitney et al. (2003) to a much larger parameter space. Observationally, Lada et al. (2006) have used IRAC and MIPS colors from Spitzer observations to classify the optical thickness of disks around pre-main-sequence stars in IC 348, using the terms "anemic disks" and "thick disks" to point to objects roughly in the Class III and Class II portions of the ubiquitous "Class system" (Lada 1987; Greene et al. 1994).

Figure 11 shows three color-color diagrams and one colormagnitude diagram for the 235 Serpens YSOs compared to the density of models from Robitaille et al. (2006) for a model cluster at the distance of Serpens with brightnesses limited to those appropriate for our sensitivity levels. For two of the diagrams the figure also indicates the approximate areas where most of the models fall for the three physical stages, I, II, and III, described by Robitaille et al. (2006) that roughly correspond to the observational "Classes" I+flat, II, and III. These diagrams display, first of all, that YSOs come in a wide range of colors and brightnesses; large areas of all the diagrams are occupied. In a general way we find objects located in these diagrams where the models of Whitney et al. (2003), Allen et al. (2004), and Robitaille et al. (2006) would predict for pre-main-sequence stars with a range of evolutionary states, as well as general agreement with the locations of young objects found in IC 348 by Lada et al. (2006). For example, consistent with our finding in Paper II that the bulk of the YSOs are Class II objects, we see a strong concentration in the area around $[5.8]-[8.0]=0.8,[3.6]-[4.5]=0.5$, where both Allen et al. (2004) and Whitney et al. (2003) predict such sources should be located. In addition, there is structure in the [8.0] versus [3.6] - [8.0] color-magnitude diagram for the distribution of Serpens YSOs that is also evident in the density of models of Robitaille et al. (2006). There are, however, some interesting differences. The "stage II" area from Robitaille et al. (2006) includes a smaller fraction of our nominal Class II objects than might be expected. There is also a larger number of very red objects in several of the diagrams than is predicted by the density of models in those areas, considering that the faintest gray levels correspond to a probability equal to $10^{-4}$ of the maximum probability. Some of this may be due to effects of reddening, but it suggests that the models may also need some refinement, despite their rough agreement with the distribution of observed colors.

These data can also be examined to see if there is any correlation between luminosity and infrared excess, e.g., the [8.0] versus [3.6] - [8.0] color-magnitude diagram. For example, if higher luminosity young objects had higher levels of multiplicity (e.g., Lada 2006), then it is possible that this would be manifested in less massive disks with perhaps large inner holes. Figure 11 shows no such correlation; in fact, if anything there appears to be a lack of relatively blue colors for the brightest objects. Figure 8, which displays the distribution of spectral slopes, and the accompanying Table 5 also show no measurable effect within the scatter for a dependence of spectral slope on luminosity.

\subsection{Spectral Energy Distribution Modeling}

In order to go beyond the general conclusions possible from color-color and color-magnitude plots, we have modeled the large fraction of our sources for which enough data are available to characterize them as likely star-plus-disk objects. For this analysis 

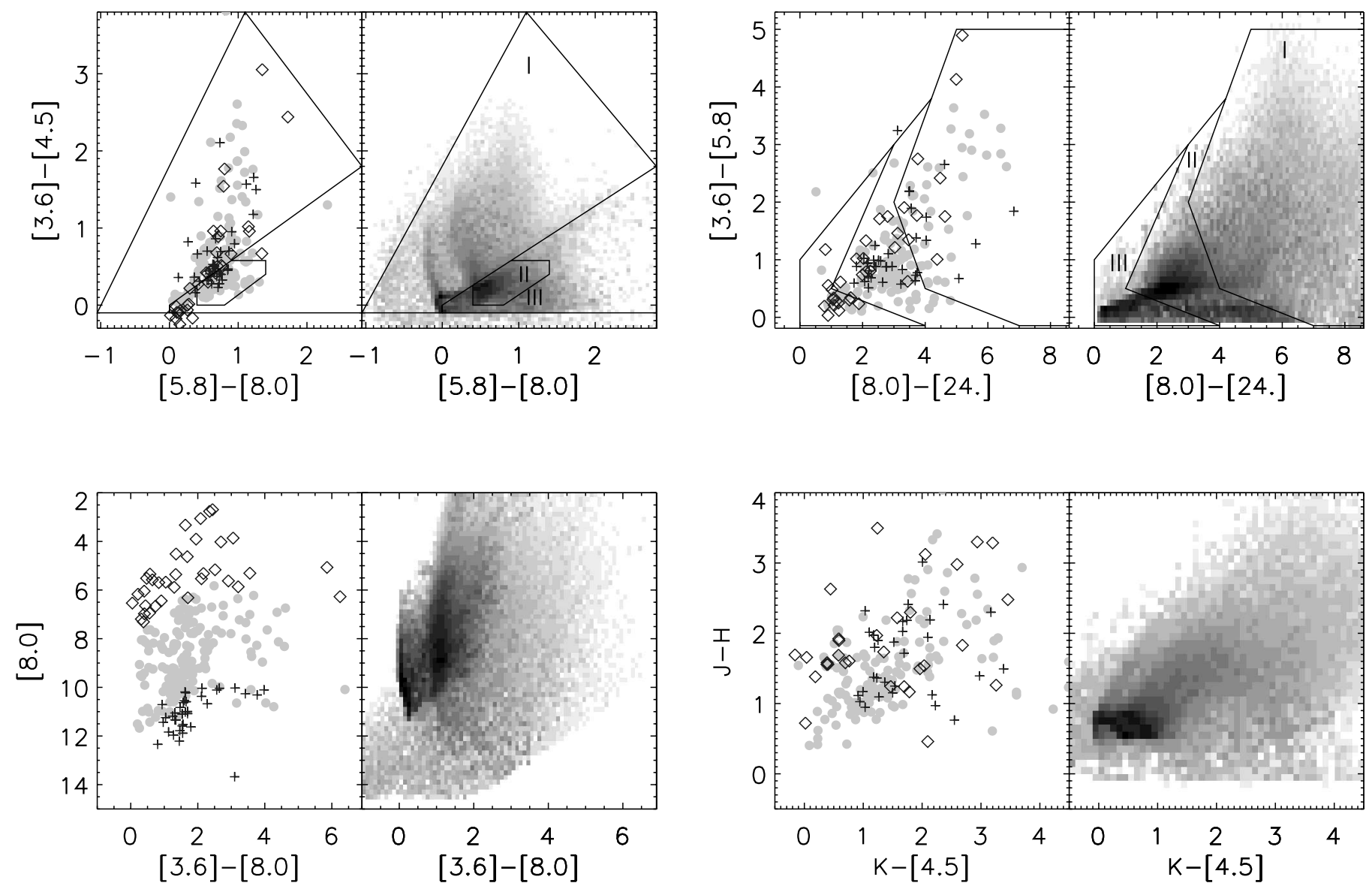

FIG. 11.-Color-color and color-magnitude plots of the 235 Serpens YSOs compared with the distributions from models of Robitaille et al. (2006). The symbols are for the three different luminosity groups discussed in the text: $L>1 L_{\odot}$ (open diamonds), $0.02 L_{\odot}<L<1 L_{\odot}$ (gray filled circles), and $L<0.02 L_{\odot}($ plus signs). The model data were those for their model cluster, with a distance of $260 \mathrm{pc}$ for Serpens and with the c2d completeness limits. Also shown are the rough areas from Robitaille et al. (2006) occupied by mainly stage I, II, and III models.

we have selected all Class II and Class III YSOs. We expect these objects to have a reddened photosphere plus an infrared excess coming from the circumstellar material, most likely in a disk configuration. For completeness we show, without modeling, the SEDs of the remaining YSOs. Figures 12-20 show the SEDs of all Class I, flat, Class II, and Class III sources. The open circles show the observed fluxes and generally include 2MASS $J, H$, and $K$ fluxes followed by the four IRAC fluxes at 3.6, 4.5, 5.8, and $8.0 \mu \mathrm{m}$ and by the MIPS fluxes at 24 and $70 \mu \mathrm{m}$ when available. For the Class II and III sources, we characterize the emission from the disk by comparing the energy distribution with that of a low-mass star. For this, we have computed the extinction from the $J-K$ colors assuming a $\mathrm{K} 7$ underlying star, and then we have dereddened the observed fluxes with the interstellar extinction law of Weingartner \& Draine (2001) with $R_{v}=5.5$ to fit the dereddened SEDs to a NEXTGEN (Hauschildt et al. 1999) model of a K7 stellar photosphere. In the plots, the filled circles show the dereddened fluxes and the gray line shows the photospheric emission from the star. Each SED is labeled with the identification number in Table 2. The parameters of the model fits are listed in Tables 7 and 8.

Two main assumptions are used in this approach. The first is that the star is a low-mass star; this is reasonable given the fact that the luminosity function discussed earlier peaks at $L \sim$ few $\times$ $10^{-2} L_{\odot}$. In addition, J. M. Oliveira et al. (2007, in preparation) have found that 30 of the 39 YSOs they observed out of our sample had spectral types of $\mathrm{K}$ or $\mathrm{M}$. The second assumption is that there is no substantial excess in the near-infrared bands, where we consider all flux to be photospheric. This assumption is obviously only correct for those stars in the sample without strong ongoing disk accretion. Cieza et al. (2005) have demonstrated that near-IR excesses as short as $J$ band can be seen in some actively accreting $\mathrm{T}$ Tauri stars, and they give an observed range of $J$-band excesses of 0.1-1.0 mag for their sample of sources in Taurus. The effect of this excess on the computed parameters implies that the stellar luminosities for the Class II objects are upper limits and therefore the disk luminosities are lower limits. More specifically, neglecting an excess flux in the $J$ band of $1.1 \mathrm{mag}$ in one object would reduce its $L_{\text {disk }} / L_{\text {star }}$ ratio by $12 \%$ and modify the disk emission SED, diminishing its excess mostly at the shorter wavelengths. That would only happen, however, to the fraction of objects that are strongly accreting. J. M. Oliveira et al. (2007, in preparation) found strong $\mathrm{H} \alpha$ emission indicative of active accretion in only 12 out of 39 Serpens YSOs observed. We ran a test removing these objects from the final statistics, and this did not change the overall picture.

The dashed line in the SED plots is the median SED of T Tauri stars in Taurus (Hartmann et al. 2005) normalized to the dereddened $J$-band flux of our SEDs. It represents the typical SED of an optically thick accreting disk around a classical T Tauri star (CTTS) and is shown here to allow a qualitative estimation of the presence of disk evolution and dust settling. Based on similarity to this median SED, we define as "T" an SED that is identical to it, within the errors, as "L" an SED with lower fluxes at some 


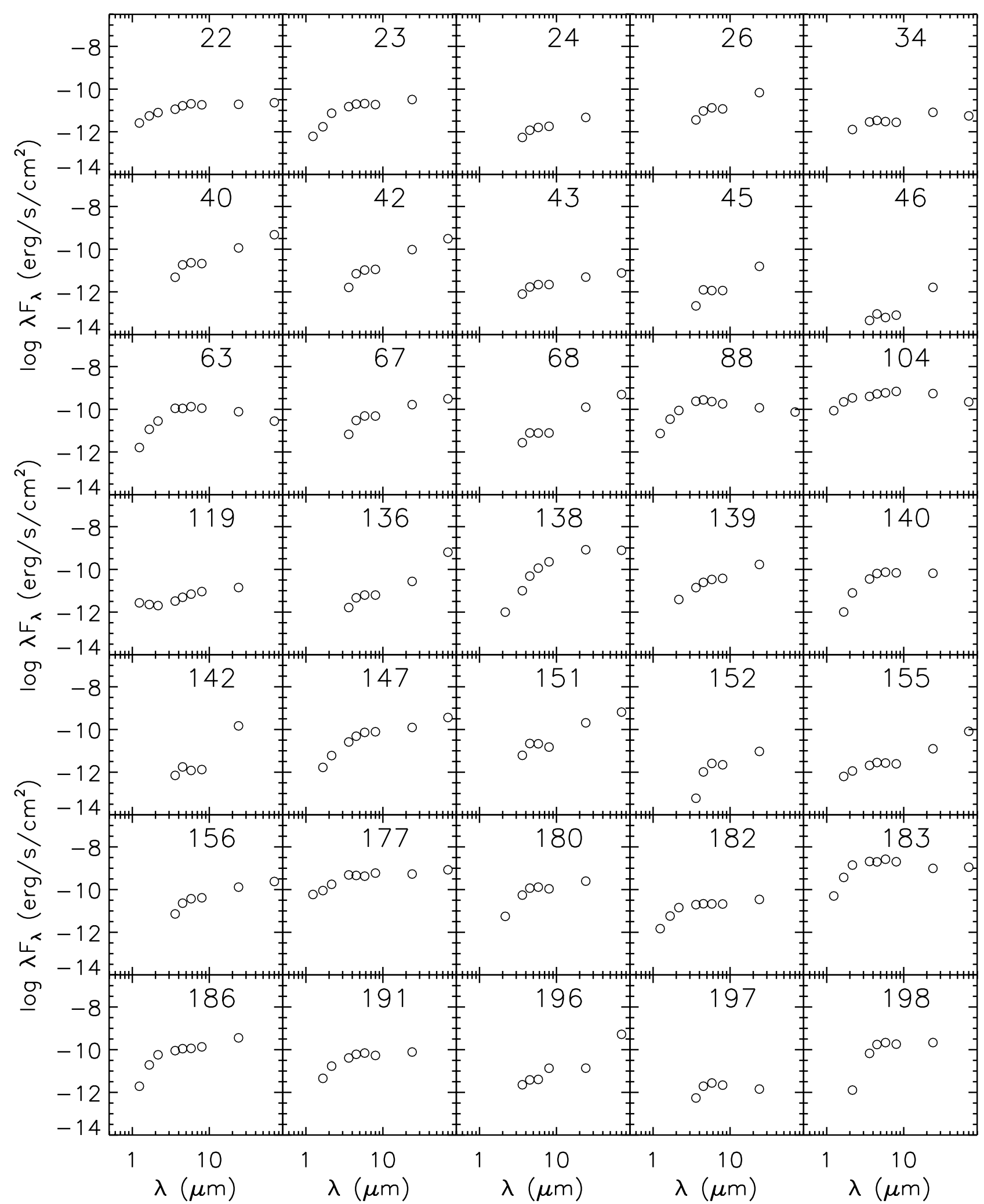

FIG. 12.- SEDs of the Class I sources in the sample. The open circles signal the observed fluxes from $J$ band to MIPS-70 when available. The label gives the index in Table 2. 


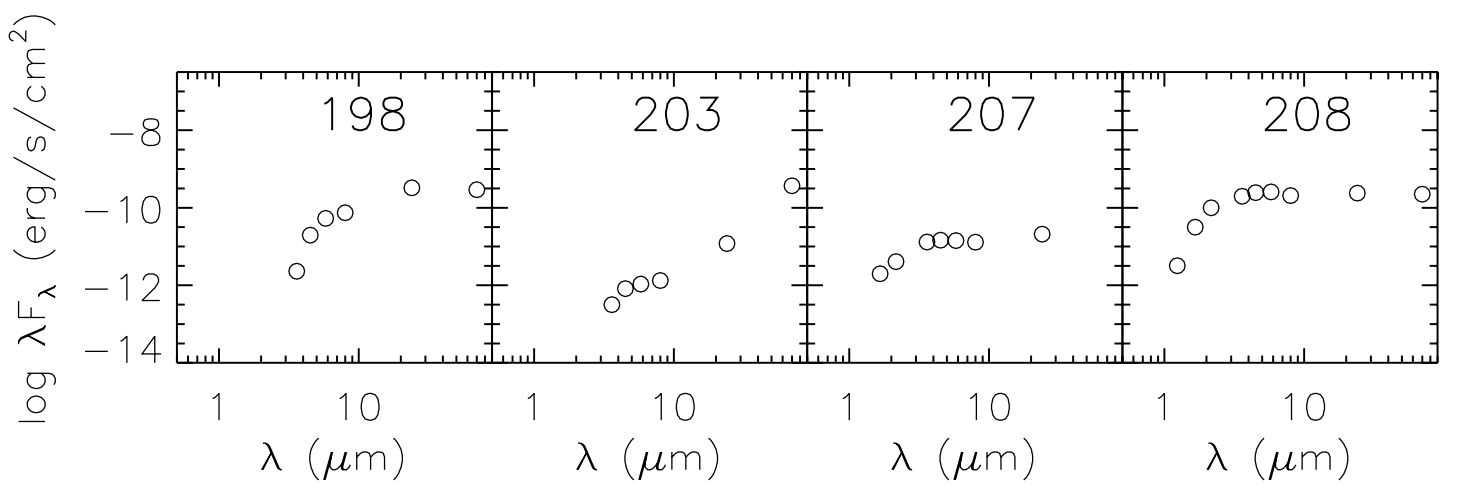

FIG. 13. - SEDs of the Class I sources in the sample (continued). Symbols as in Fig. 12.

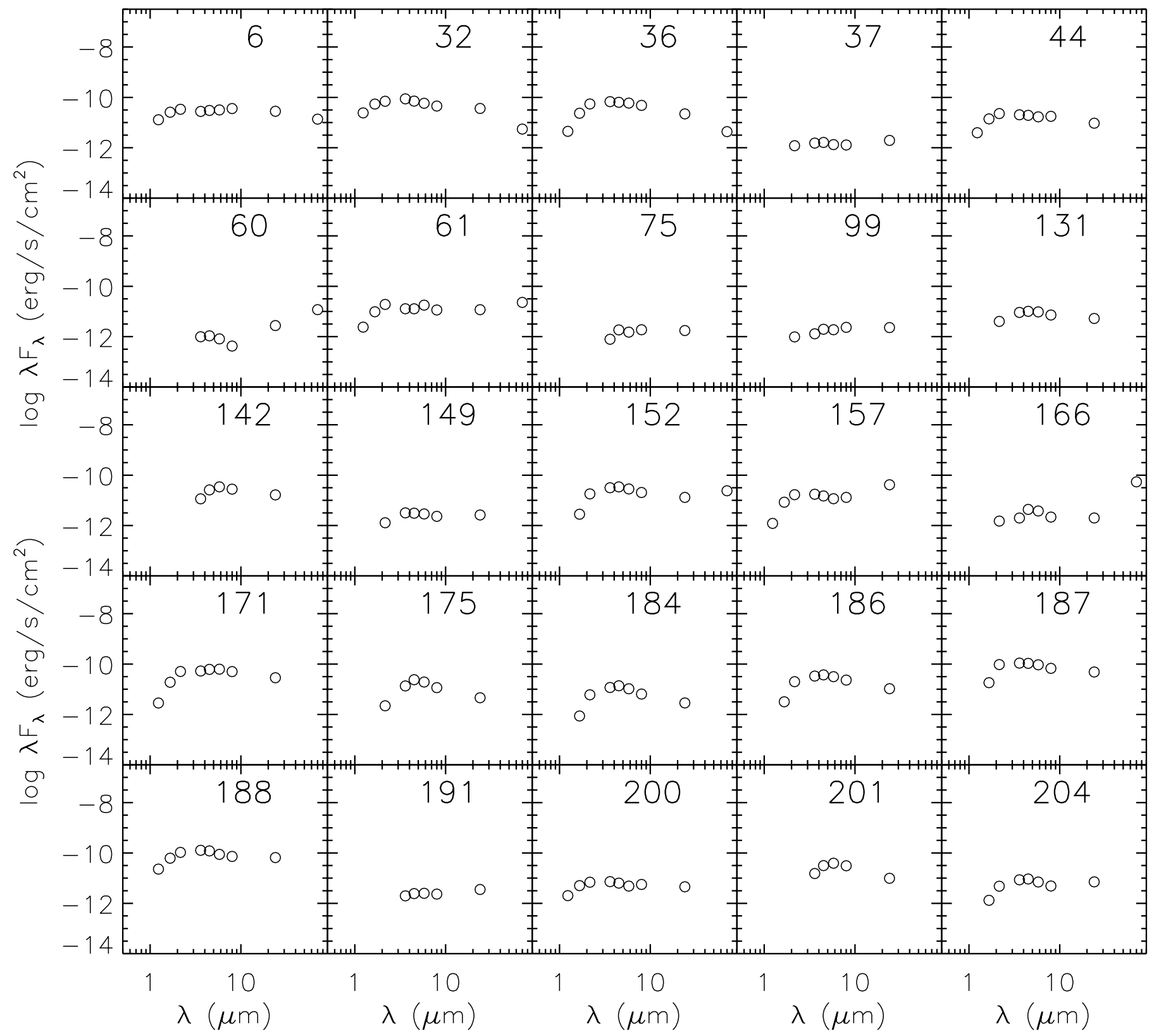

FIG. 14.-SEDs of the flat sources in the sample. Symbols as in Fig. 12. 


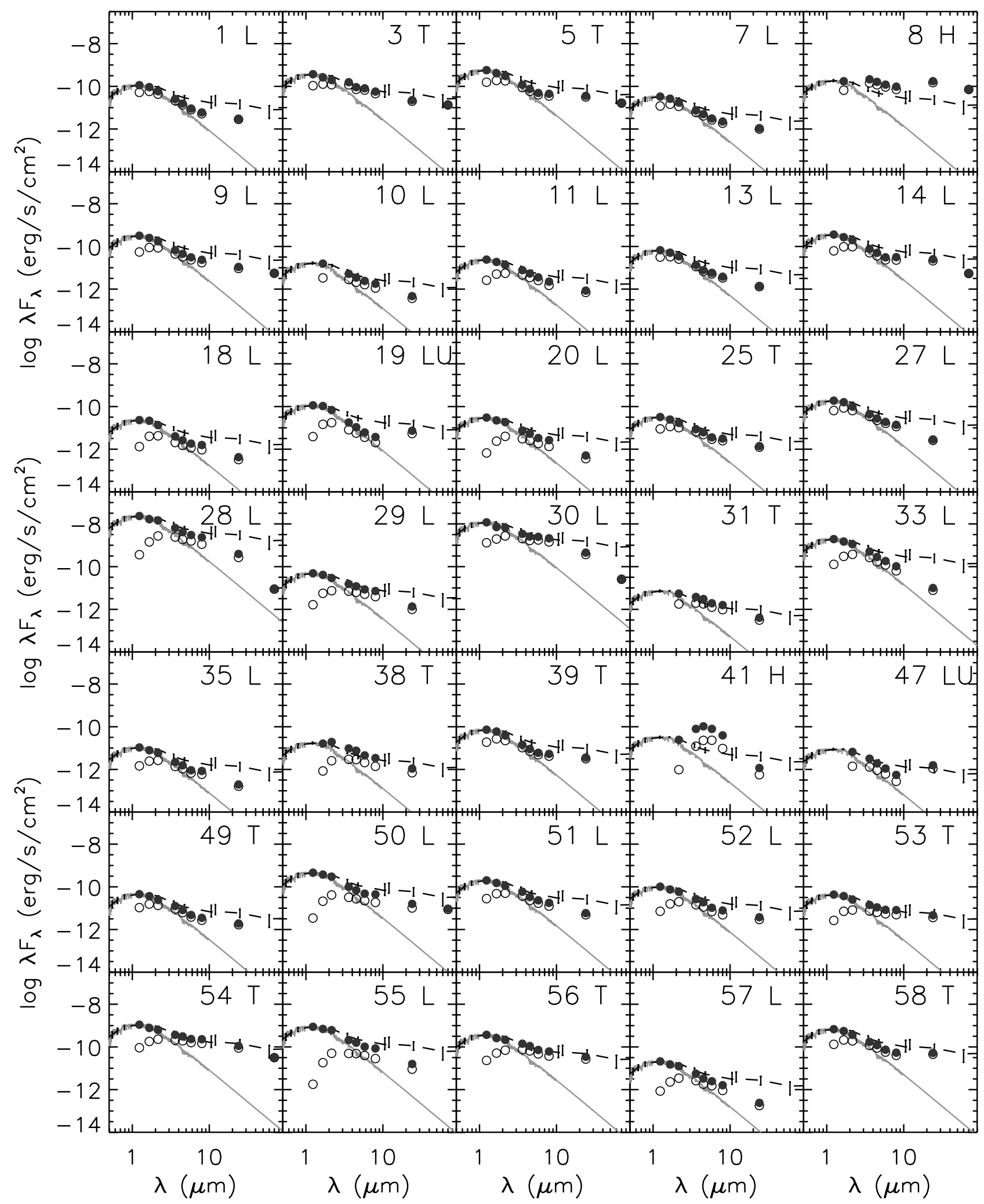

FIG. 15.-SEDs of the Class II sources in the sample. The open and filled circles show the observed and dereddened fluxes, respectively. The gray line shows the stellar model of a K7 star, and the dashed line shows the median SED of the T Tauri stars in Taurus by Hartmann et al. (2005) normalized to the dereddened $J$-band flux for comparison. See text for more information. 


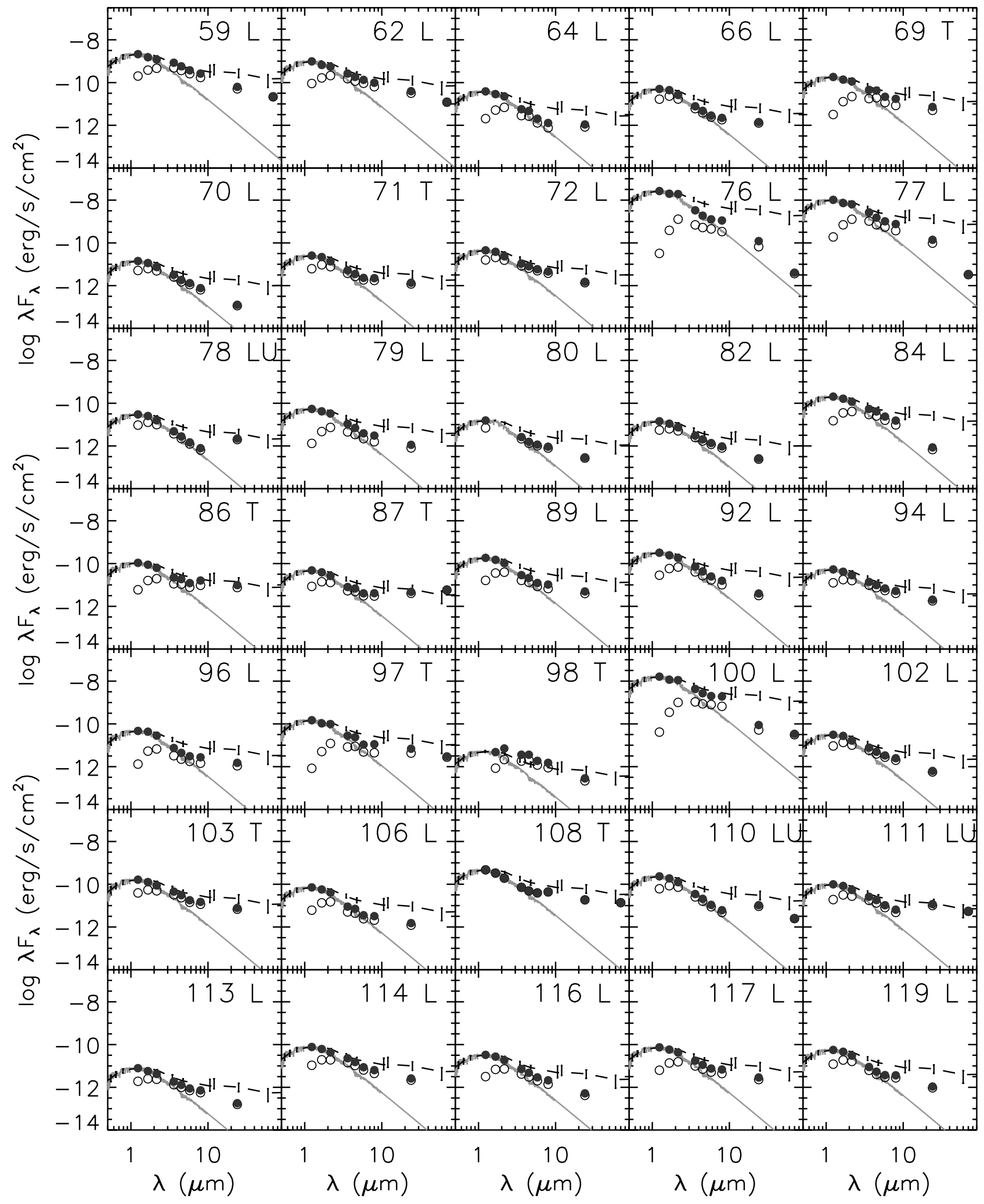

FIG. 16. - SEDs of the Class II sources in the sample (continued). 


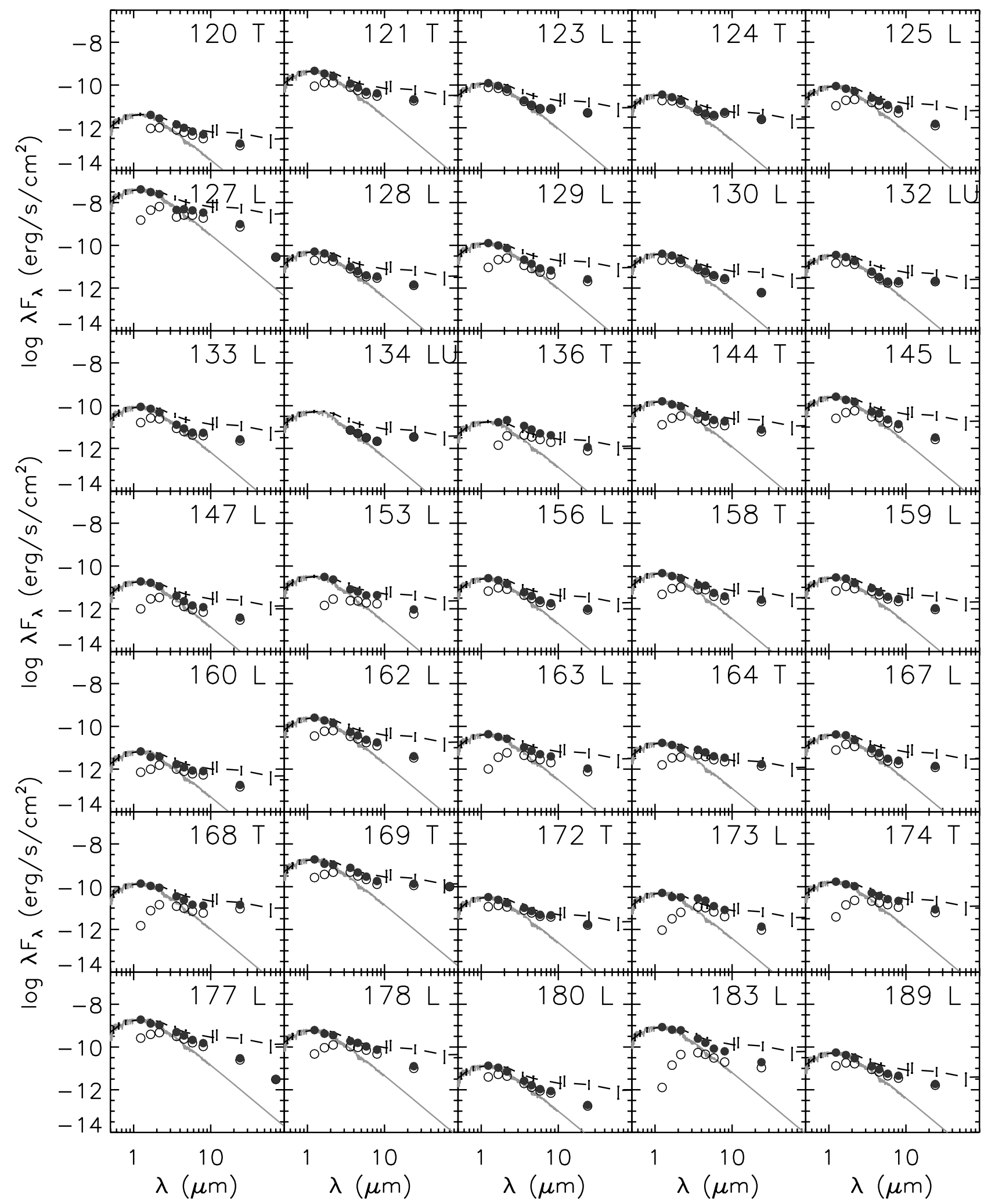

FIG. 17.-SEDs of the Class II sources in the sample (continued). 


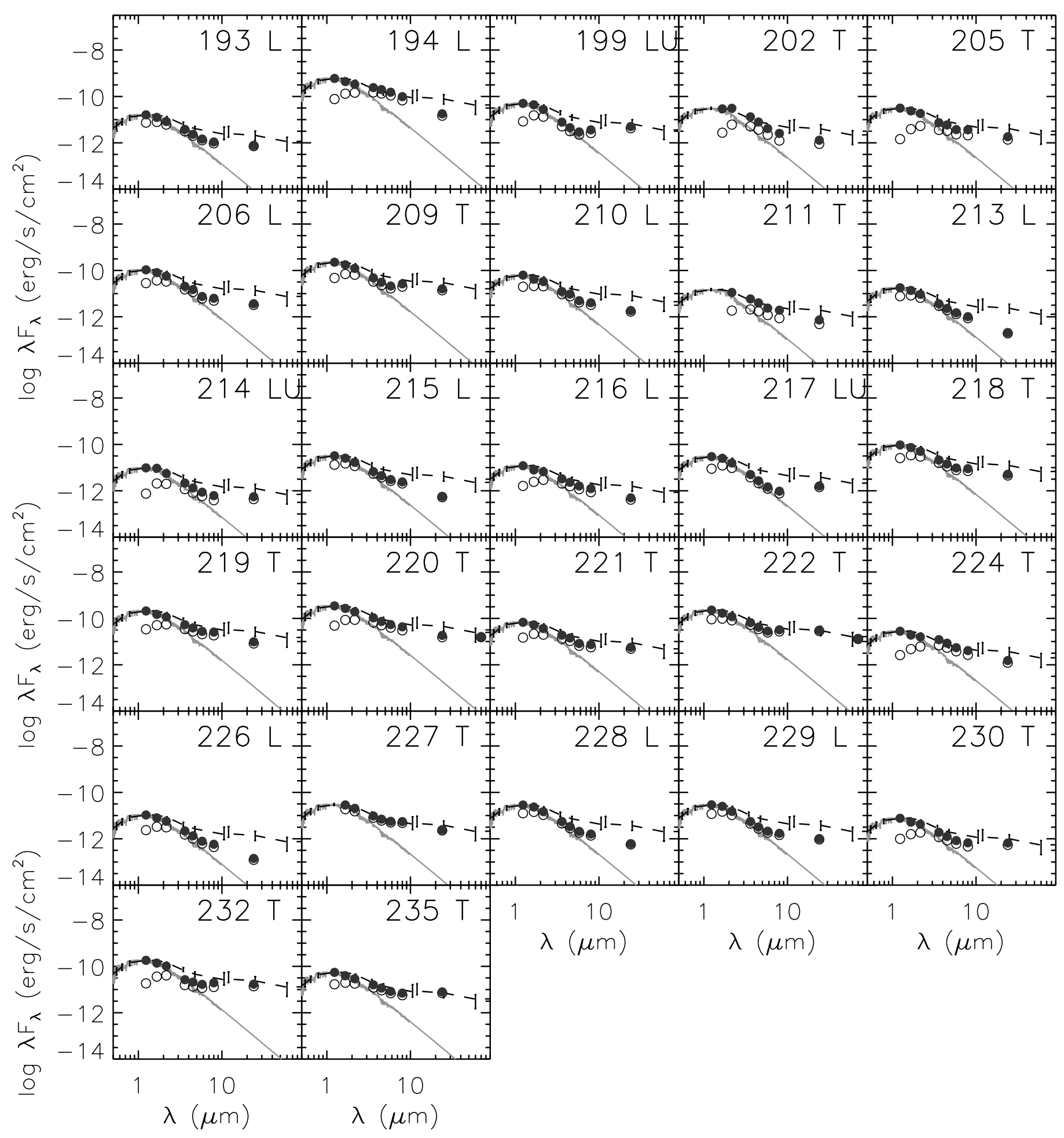

FIG. 18. - SEDs of the Class II sources in the sample (continued).

wavelengths, and as " $\mathrm{H}$ " an object with larger fluxes than the median T Tauri SED. We have labeled the objects with these codes and use them to interpret the state of disk evolution. We interpret the L-type objects as thin disks that could perhaps result from dust grain growth and settling to the midplane, similar to the anemic disks in Lada et al. (2006). Objects 3 and 5 are good examples of T-type objects, numbers 11 and 18 are L-type stars, and number 8 is an H-type object. Also, we have labeled as "LU" objects that have photospheric fluxes up to around $8 \mu \mathrm{m}$ but then a sudden jump at longer wavelengths to the levels of a
T Tauri disk. We believe that these objects may have large inner holes in their still optically thick disks; number 19 is one example of these.

Out of the 171 sources in the sample that are Class II or Class III, $44(26 \%)$ are $\mathrm{T}$ type and so equivalent to a CTTS, $125(73 \%)$ are L type and so show evidence of some degree of disk evolution, and finally $2(1 \%)$ show larger fluxes than the median SED of Taurus. The latter category is generally the result of imperfect fitting to the stellar photosphere due to lack of near-IR data and will not be considered further. 


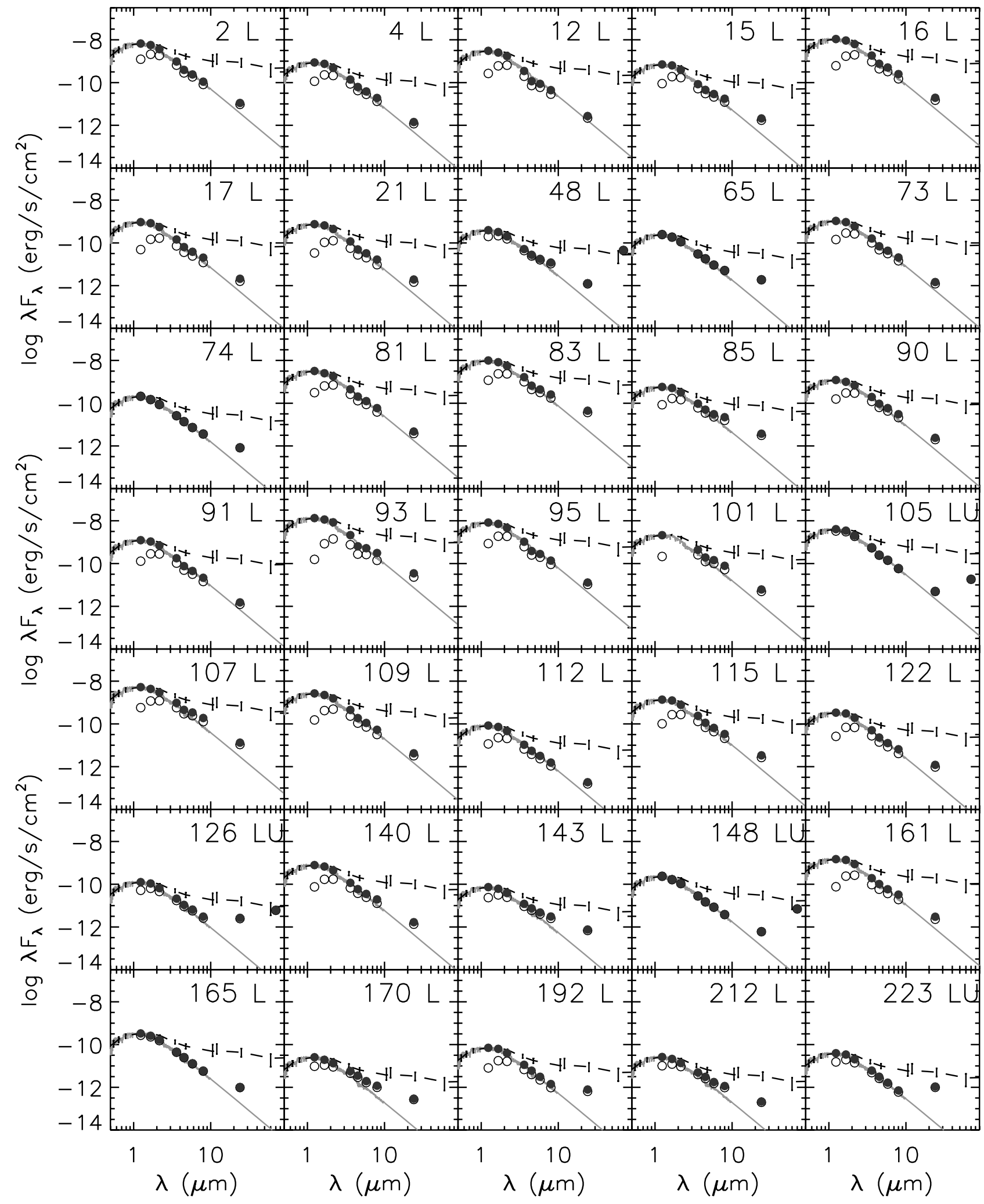

FIG. 19.-SEDs of the Class III sources in the sample. 


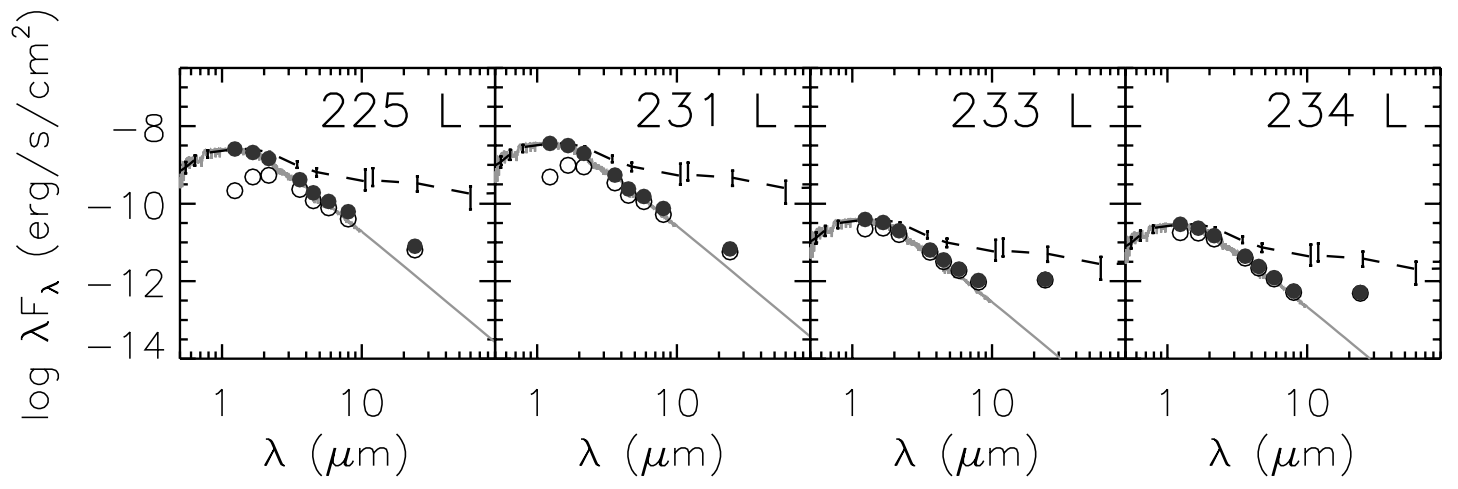

FIG. 20.- SEDs of the Class III sources in the sample (continued).

The numbers above indicate that there are many young stars in Serpens with disks that are virtually identical to those in Taurus. This points to a very similar origin of the disk structure across star-forming regions. Within the uncertainties in our modeling process discussed above, however, we see a majority of L-type objects $(73 \%)$ in Serpens. This suggests that the disks in Serpens may be generally more evolved or flattened than those in Taurus. Also, interestingly the number of LU-type objects, or those with large inner holes, amounts to 14 (11\% of the L-type objects and only $6 \%$ of the total YSO sample including Class I and flat sources). This very small incidence indicates that either the process producing these large inner holes is a very fast transitional phase in the disk evolution or only a small number of systems undergo that phase. We examine this issue below. In order to put firmer limits on these numbers, it will be necessary to get optical spectral types for the stars and optical photometry in order to produce more accurate photosphere/disk/extinction models.

The SEDs also allow a rough study of the disk properties across the surveyed area. For this purpose we integrated the stellar and total fluxes to calculate a ratio between the stellar and disk fluxes. The corresponding distribution is shown in Figure 21. Again, the solid line is for the whole Class II and III sample, and the dotted line corresponds to the T-type SEDs. To guide the eye, we have marked the approximate regimes of these ratios measured in debris disks $\left(L_{\text {disk }} / L_{\text {star }}<0.02\right)$, passive disks $\left(0.02<L_{\text {disk }} / L_{\text {star }}<\right.$ 0.08 ; Kenyon \& Hartmann 1987), and accretion disks $\left(L_{\text {disk }} / L_{\text {star }}>\right.$ $0.1)$, respectively. The figure illustrates well the large variety of disk evolutionary phases that we observe in Serpens, as well as the large majority of massive, accreting disks in the region.

TABLE 7

Disk Modeling Results in Class II Sources

\begin{tabular}{|c|c|c|c|c|c|c|}
\hline Star & SED Type & $A_{V}$ & $L_{\text {star }}$ & $L_{\mathrm{disk}} / L_{\mathrm{star}}$ & $\lambda_{\text {turnoff }}$ & $\alpha_{\text {excess }}$ \\
\hline $1 \ldots \ldots \ldots$ & $\mathrm{L}$ & 2.9 & 0.33 & 0.088 & 5.8 & -0.7 \\
\hline 3................... & $\mathrm{T}$ & 4.6 & 1.06 & 0.330 & 2.2 & -0.8 \\
\hline $5 \ldots \ldots \ldots \ldots$ & $\mathrm{T}$ & 4.9 & 1.64 & 0.165 & 3.6 & -0.5 \\
\hline $7 \ldots \ldots \ldots \ldots \ldots$ & $\mathrm{L}$ & 3.8 & 0.09 & 0.110 & 3.6 & -1.0 \\
\hline 9..................... & $\mathrm{L}$ & 6.6 & 0.91 & 0.141 & 3.6 & -0.8 \\
\hline $10 \ldots \ldots \ldots \ldots$ & $\mathrm{L}$ & 9.9 & 0.05 & 0.190 & 3.6 & -1.3 \\
\hline $11 \ldots \ldots \ldots$ & $\mathrm{L}$ & 8.4 & 0.07 & 0.186 & 2.2 & -1.2 \\
\hline $13 \ldots \ldots \ldots \ldots$ & $\mathrm{L}$ & 2.8 & 0.19 & 0.084 & 5.8 & -1.0 \\
\hline $14 \ldots \ldots \ldots \ldots$ & $\mathrm{L}$ & 6.6 & 1.03 & 0.182 & 3.6 & -0.7 \\
\hline $18 \ldots \ldots \ldots \ldots$ & $\mathrm{L}$ & 10.8 & 0.07 & 0.098 & 3.6 & -1.1 \\
\hline
\end{tabular}

Notes.-Table 7 is published in its entirety in the electronic edition of the Astrophysical Journal. A portion is shown here for guidance regarding its form and content.
In order to study the disk evolution in the cloud, there is need for a better characterization of the full sample with optical spectroscopy. That work is in progress and will be published elsewhere. Here we explore further the shapes of the SEDs to test another evolutionary diagnostic of the disk status. We have found that the more commonly used evolutionary metrics, e.g., spectral

TABLE 8

Disk Modeling Results in Class III Sources

\begin{tabular}{|c|c|c|c|c|c|c|}
\hline Star & SED Type & $A_{V}$ & $L_{\text {star }}$ & $L_{\text {disk }} / L_{\text {star }}$ & $\lambda_{\text {turnoff }}$ & $\alpha_{\text {excess }}$ \\
\hline $2 \ldots$ & $\mathrm{L}$ & 6.3 & 19.19 & 0.013 & 24.0 & -99.0 \\
\hline 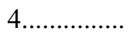 & $\mathrm{L}$ & 7.6 & 2.50 & 0.028 & 24.0 & -99.0 \\
\hline $12 \ldots \ldots \ldots \ldots$ & $\mathrm{L}$ & 9.1 & 8.76 & 0.015 & 24.0 & -99.0 \\
\hline $15 \ldots$ & $\mathrm{L}$ & 7.7 & 2.02 & 0.025 & 8.0 & -1.9 \\
\hline ......... & $\mathrm{L}$ & 10.8 & 31.18 & 0.041 & 8.0 & -2.3 \\
\hline $17 \ldots \ldots \ldots \ldots$ & $\mathrm{L}$ & 11.1 & 2.69 & 0.036 & 8.0 & -2.1 \\
\hline $21 \ldots$ & $\mathrm{L}$ & 11.7 & 2.16 & 0.038 & 8.0 & -2.0 \\
\hline $48 \ldots$ & $\mathrm{L}$ & 2.5 & 1.13 & 0.054 & 8.0 & 0.6 \\
\hline $65 \ldots \ldots \ldots \ldots$ & $\mathrm{L}$ & 0.0 & 0.70 & 0.016 & 8.0 & -0.9 \\
\hline $73 \ldots \ldots \ldots . .$. & $\mathrm{L}$ & 7.5 & 3.06 & 0.022 & 24.0 & -99.0 \\
\hline $74 \ldots \ldots \ldots \ldots$ & $\mathrm{L}$ & 0.0 & 0.60 & 0.010 & 8.0 & -1.3 \\
\hline $81 \ldots \ldots \ldots . . .$. & $\mathrm{L}$ & 8.7 & 9.23 & 0.021 & 24.0 & -99.0 \\
\hline $83 \ldots \ldots \ldots . . .$. & $\mathrm{L}$ & 8.0 & 28.57 & 0.034 & 8.0 & -1.6 \\
\hline $85 \ldots \ldots \ldots \ldots$ & $\mathrm{L}$ & 7.2 & 1.66 & 0.048 & 5.8 & -1.5 \\
\hline $90 \ldots \ldots \ldots \ldots$ & $\mathrm{L}$ & 7.7 & 3.50 & 0.034 & 8.0 & -2.3 \\
\hline $91 \ldots \ldots \ldots . . . . . . .$. & $\mathrm{L}$ & 8.5 & 3.55 & 0.020 & 24.0 & -99.0 \\
\hline $93 \ldots \ldots \ldots . . . . .$. & $\mathrm{L}$ & 16.7 & 38.30 & 0.051 & 8.0 & -2.2 \\
\hline $95 \ldots \ldots \ldots \ldots$ & $\mathrm{L}$ & 8.5 & 23.79 & 0.015 & 24.0 & -99.0 \\
\hline $101 \ldots \ldots \ldots . .$. & $\mathrm{L}$ & 8.6 & 6.09 & 0.074 & 5.8 & -2.2 \\
\hline $105 \ldots \ldots \ldots$ & LU & 0.7 & 11.61 & 0.010 & 24.0 & 1.2 \\
\hline $107 \ldots \ldots \ldots . .$. & $\mathrm{L}$ & 8.3 & 15.02 & 0.055 & 5.8 & -2.3 \\
\hline $109 \ldots \ldots \ldots$ & $\mathrm{L}$ & 10.7 & 7.62 & 0.037 & 8.0 & -2.3 \\
\hline $112 \ldots \ldots \ldots$ & $\mathrm{L}$ & 7.4 & 0.24 & 0.018 & 8.0 & -1.9 \\
\hline $115 \ldots \ldots \ldots$ & $\mathrm{L}$ & 9.7 & 3.90 & 0.044 & 8.0 & -2.1 \\
\hline $122 \ldots \ldots \ldots$ & $\mathrm{L}$ & 9.6 & 0.96 & 0.028 & 8.0 & -1.5 \\
\hline $126 \ldots \ldots \ldots$ & LU & 3.3 & 0.36 & 0.062 & 8.0 & 0.3 \\
\hline $140 \ldots \ldots \ldots . .$. & $\mathrm{L}$ & 8.8 & 2.25 & 0.032 & 8.0 & -2.2 \\
\hline $143 \ldots \ldots \ldots$ & $\mathrm{L}$ & 4.2 & 0.21 & 0.052 & 5.8 & -1.3 \\
\hline $148 \ldots \ldots \ldots$ & LU & 0.0 & 0.67 & 0.017 & 24.0 & 2.3 \\
\hline $161 \ldots \ldots \ldots . .$. & $\mathrm{L}$ & 11.2 & 4.26 & 0.032 & 8.0 & -2.2 \\
\hline $165 \ldots \ldots \ldots$ & $\mathrm{L}$ & 0.7 & 0.94 & 0.014 & 8.0 & -1.6 \\
\hline $170 \ldots \ldots \ldots$ & $\mathrm{L}$ & 3.7 & 0.07 & 0.079 & 5.8 & -1.3 \\
\hline $192 \ldots \ldots \ldots$ & $\mathrm{L}$ & 8.1 & 0.20 & 0.034 & 8.0 & -0.5 \\
\hline $212 \ldots \ldots \ldots$ & $\mathrm{L}$ & 3.5 & 0.07 & 0.062 & 5.8 & -1.5 \\
\hline $223 \ldots \ldots \ldots$ & LU & 3.5 & 0.11 & 0.025 & 8.0 & 0.4 \\
\hline $225 \ldots \ldots \ldots$ & $\mathrm{L}$ & 9.3 & 7.38 & 0.036 & 8.0 & -1.9 \\
\hline $231 \ldots \ldots \ldots$ & $\mathrm{L}$ & 7.5 & 10.29 & 0.025 & 8.0 & -2.2 \\
\hline $233 \ldots \ldots \ldots$ & $\mathrm{L}$ & 2.1 & 0.11 & 0.038 & 8.0 & 0.1 \\
\hline $234 \ldots \ldots \ldots \ldots$ & $\mathrm{L}$ & 1.9 & 0.08 & 0.022 & 8.0 & -0.1 \\
\hline
\end{tabular}




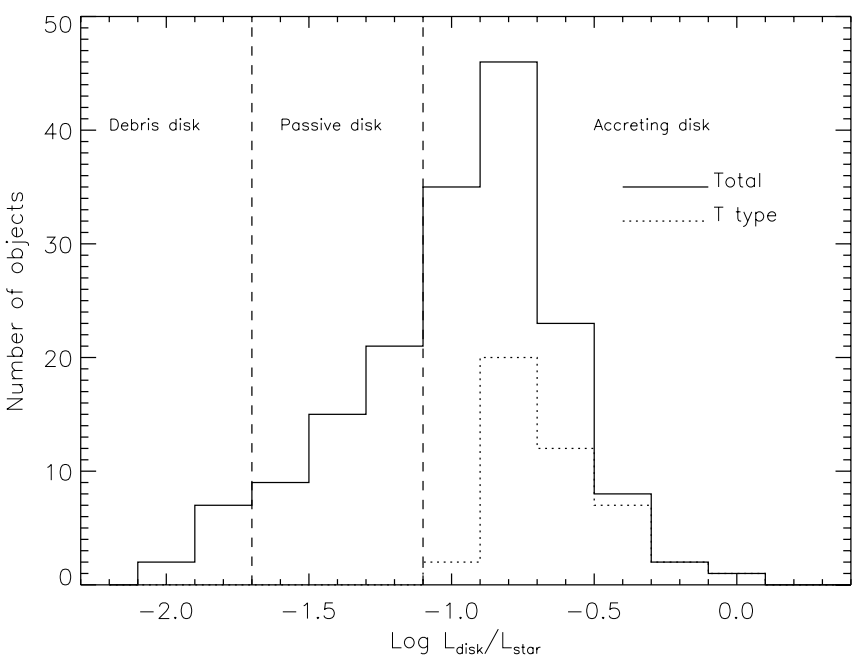

FIG. 21.-Distribution of disk-to-star luminosity ratios. The solid and dashed histograms are for the total sample and T Tauri-like sample of SEDs, respectively. Also marked are the typical ranges of $L_{\text {disk }} / L_{\text {star }}$ ratios for debris disks, passive irradiated disks, and accretion disks. The figure indicates that objects of all three evolutionary stages are found in Serpens, with a predominance of young accreting T Tauri-type stars.

slope $\alpha$, bolometric temperature $T_{\text {bol }}$, or the Class system discussed earlier, do not seem to capture the full range of phenomena apparent in our distribution of SEDs for YSOs. We therefore use $\alpha_{\text {excess }}$ and $\lambda_{\text {excess }}$, two new second-order SED parameters presented in Cieza et al. (2007) that will be discussed in more detail in a separate paper. In short, $\lambda_{\text {excess }}$ is the last wavelength where the observed flux is photospheric and $\alpha_{\text {excess }}$ is the slope computed as $d \log \left(\lambda F_{\lambda}\right) / d \log \lambda$ starting from $\lambda_{\text {excess. }}$. The first parameter gives us an indication of how close the circumstellar matter is to the central object, and the latter one a measure of how optically thick it is. Given the assumptions above for the fitting process, our values are upper limits for $\lambda_{\text {excess }}$ and correspondingly lower limits for $\alpha_{\text {excess }}$ for the Class II sources. We assume good stellar fits for the Class III sources.

Figure 22 shows both values for a sample of CTTSs from Cieza et al. (2005), weak-lined T Tauri stars (WTTSs) from Cieza et al. (2007), debris disks from Chen et al. (2005), and our sample of YSOs in Serpens. Cieza et al. (2007) found that $\lambda_{\text {excess }}$ is well correlated with evolutionary phase and also, as seen in Figure 22, that $\alpha$ is observed over wider ranges for later evolutionary phases. This suggests a large range of possible evolutionary paths for circumstellar disks. Objects could evolve to the lower right side of the diagram, which could be interpreted as a sign of dust grain growth and settling to the disk midplane; they could also move to the right and up, from creation of a large inner hole in their disks while retaining a massive outer disk, and also, as this diagram shows, all intermediate pathways in between both extremes.

\section{SELECTED SOURCES}

\subsection{The Coldest Objects}

Table 9 lists the 15 YSOs that display the coldest energy distributions. These are the objects with a ratio of $F_{70} / F_{24}>8$. Six of these are in cluster A (the core) and four in cluster B. Two are in a small grouping about $0.2^{\circ}$ northeast of cluster $B$, and the remaining three appear as isolated cold objects, although in areas with other moderately red objects. All of these except for two of the isolated cold objects, IDs 105 and 148, are associated with dense clumps of millimeter emission as seen by Enoch et al.

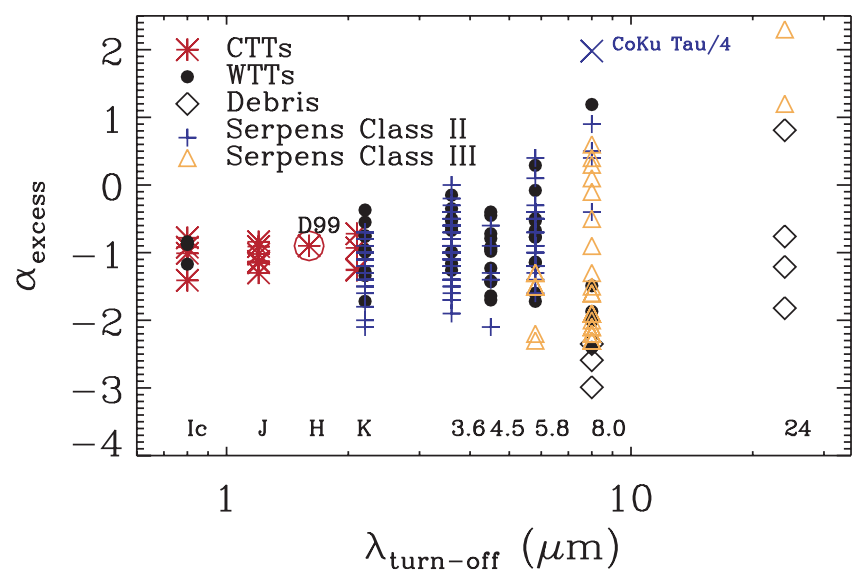

FIG. 22.-Distribution of excess slopes $\alpha_{\text {excess }}$ with respect to the wavelength at which the infrared excess begins $\lambda_{\text {turnoff }}$ for the sample of WTTSs ( filled circles), a sample of CTTSs in Lupus from Cieza et al. (2007), the median SED of CTTSs in Taurus from D'Alessio et al. (1999) in asterisks (marked as D99), and a sample of debris disks from Chen et al. (2005) in diamonds. The plus signs represent the limits for the Class II objects in Serpens, and the triangles the Class III sources. The diagram shows a much larger spread in inner disk morphologies in the more evolved objects than in the least evolved ones. The Serpens objects follow the previously observed trend.

(2006b). As discussed in the following section, two in cluster A and one in cluster B are associated with high-velocity outflows. As has been noted already by Padgett et al. (2004) and Rebull et al. (2007), it is striking how many of these coldest, most obscured YSOs are located in compact clusters together with objects that are substantially more evolved in the nominal system of classes, e.g., Class II and III.

\subsection{Outflow Sources}

A number of recent Spitzer studies have found that highvelocity shocked outflows from young stars are visible in IRAC images, typically strongest at $4.5 \mu \mathrm{m}$ (Noreiga-Crespo et al. 2004). We have examined our images for such outflows, as well as for correspondence with the lists of published $\mathrm{HH}$ objects in the region (Ziener \& Eisloffel 1999). Table 10 summarizes the results of this effort. Two strong, obvious jetlike features are seen in cluster A and one in cluster B. In addition, as shown in Table 10, we find small extended features at the positions of most of the known HH objects in Serpens that were within our covered area. The two "jets" in cluster A appear to originate from two of the most deeply embedded objects in this cluster that are associated with the submillimeter sources SMM 1 and SMM 5 (Testi \& Sargent 1998). Both of these outflows are aligned roughly in the northwest-southeast direction and are visible on both sides of the central $24 \mu \mathrm{m}$ likely exciting sources. In cluster B there is one obvious jetlike feature extending mostly south of YSO 75. There is also probably faint emission visible $30^{\prime \prime}$ to the north of the embedded source. Harvey et al. (2007) discuss the cluster B jet and several nearby Spitzer sources in more detail. Figures 23 and 24 show three-color images of clusters A and B with the color tables chosen to make these jets most visible. In addition to the optical HH objects in the Serpens clouds, there are a number of high-velocity molecular outflows that have been mapped in cluster A (Davis et al. 1999). These maps present a confusing picture of outflows, and because of the relatively larger beamwidth of the millimeter observations and close packing of infrared sources in this cluster, it is very difficult to associate the radio outflows unequivocally with particular Spitzer sources. 
TABLE 9

The Coldest YSOs $\left(F_{70} / F_{24}>8\right)$

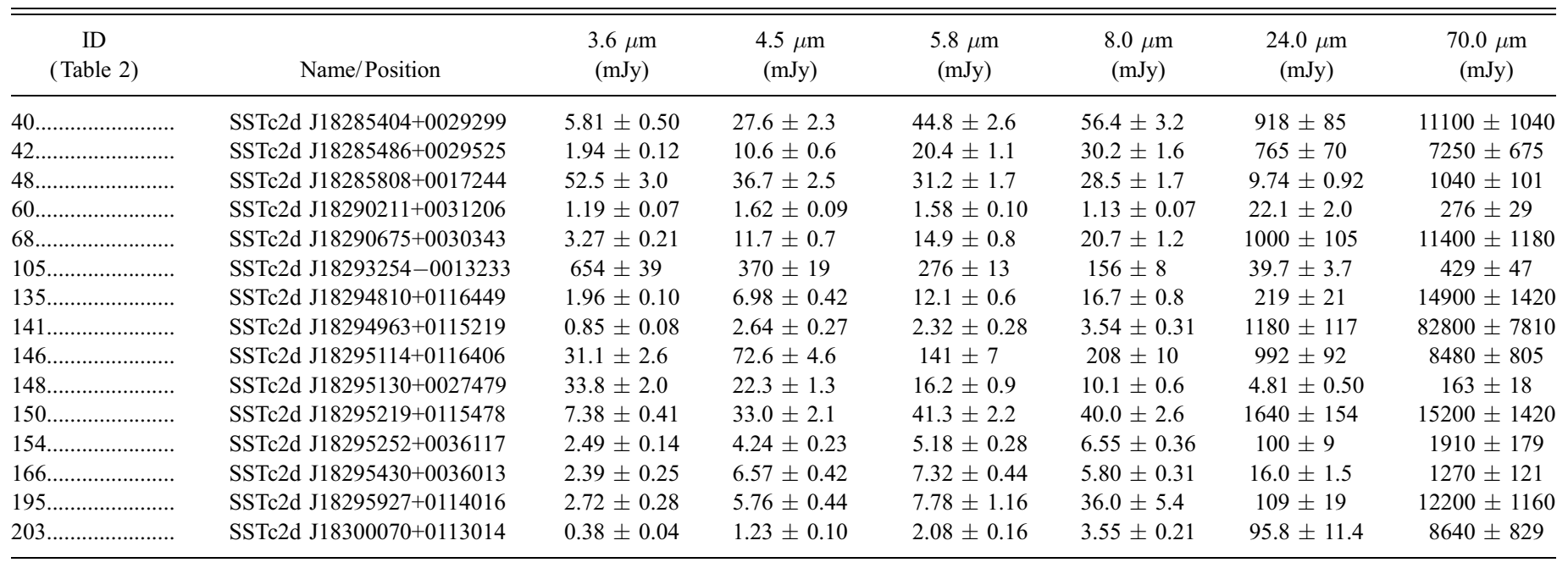

\subsection{Other Objects}

Horrobin et al. (1997) reported on the disappearance of a bright near-infrared source in Serpens from the earlier study of EC 92. Table 4 shows that we also see no obvious source at the position of EC 92-81, other than a low-S/N single-band detection of a source moderately distant from the nominal position. Interestingly, however, this area marked in Figure 23 appears to contain several small knots of emission that may represent shocked gas. For example, there are two small knots visible in our 4.5 and $8.0 \mu \mathrm{m}$ images that have no 3.6 or $24 \mu \mathrm{m}$ counterparts (and therefore not classified as YSOs). These two knots, at R.A. $=18^{\mathrm{h}} 29^{\mathrm{m}} 56.7^{\mathrm{s}}, \mathrm{decl} .=+01^{\circ} 13^{\prime} 19^{\prime \prime}(\mathrm{J} 2000.0)$, are only $12^{\prime \prime}$ south of the position of EC 92-81. Therefore, it is possible that the original source was a small clump of excited gas that has moved or dissipated since the original study of EC 92, and perhaps these Spitzer knots are related to that earlier source in some way.

\section{OVERALL RESULTS ON STAR FORMATION}

The overall picture of star formation in Serpens is summarized in Table 6. As noted earlier, the surface density of young stars is much higher in clusters A and B than in the rest of the cloud, by factors of 10-20. On the other hand, the majority of YSOs (74\%) are not in the clusters, but in the rest of the cloud. If we assume a mean mass for the stars of $0.5 M_{\odot}$ and assume that star formation has been proceeding for $2 \mathrm{Myr}$, we can estimate the rate at which the clusters and the whole cloud are converting mass into stars. These values are also given in Table 6 . The Serpens cloud that we have surveyed is turning nearly $60 M_{\odot}$ into stars per Myr. The rates for the clusters are probably underestimates because they are probably younger than $2 \mathrm{Myr}$, a typical duration for the Class II SED. Of course, a significant number of the older objects outside the clusters may have, in fact, formed in these or earlier clusters. A $1 \mathrm{~km} \mathrm{~s}^{-1}$ random motion of a YSO relative to its birthplace results in $1 \mathrm{pc}$ of movement in $10^{6} \mathrm{yr}$, or nearly $0.25^{\circ}$ at the distance of Serpens.

The distribution of YSOs over class supports the younger age for the clusters than for star formation in general in Serpens. While the ratio of the number of Class I and flat-spectrum sources to the number of Class II and Class III sources is 0.37 for the whole cloud, similar to other clouds surveyed by c2d (N. J. Evans et al. 2007, in preparation), the same ratio is 3.0 for cluster A and 1.4 for cluster B. These high ratios strongly suggest that cluster A is too young for most YSOs to have reached the Class II stage. In contrast, this ratio is 0.14 for the rest of the cloud, outside the

TABLE 10

Probable High-Velocity Outflows in Serpens

\begin{tabular}{ccccc}
\hline \hline YSO ID & $\begin{array}{c}\text { R.A. } \\
\text { (J2000.0) }\end{array}$ & $\begin{array}{c}\text { Decl. } \\
\text { (J2000.0) }\end{array}$ & Matching Name & \multicolumn{1}{c}{ Comments } \\
\hline & 182918.8 & +011415 & HH 106A, B, E & \\
& 182947.7 & +012552 & HH 107A & \\
& 183022.7 & +011618 & HH 455A & \\
& 183022.6 & +011605 & HH 455B, D & \\
& 183002.6 & +011445 & HH 459A, B & \\
141 & 182938.5 & +011826 & HH 460A, B, C, D & \\
146 & 182956.6 & +011537 & HH 478A, B & Jets to NW and SE \\
75 & 182949.6 & +011521 & SMM 1 & Jets to NW and SE \\
\hline
\end{tabular}

NotE.-Units of right ascension are hours, minutes, and seconds, and units of declination are degrees, arcminutes, and arcseconds. 


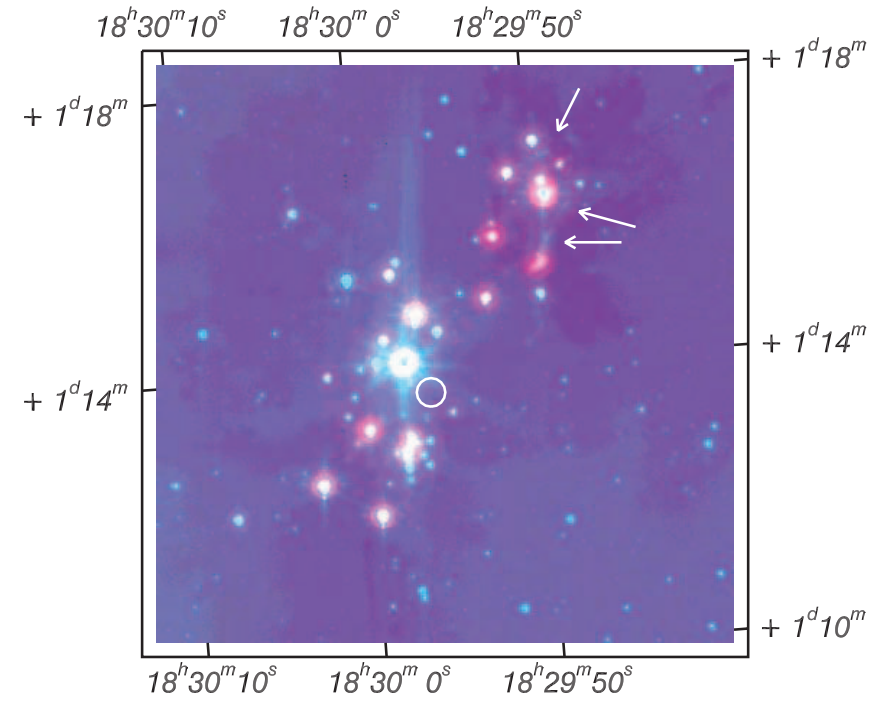

FIG. 23.- Three-color image of the cluster A area of Serpens. The color coding is blue, green, and red for $4.5,8.0$, and $24 \mu \mathrm{m}$, respectively. Some of the more obvious objects that are likely to be jets are marked by arrows. The region of the disappearing source 81 of Eiroa \& Casali (1992) is circled.

clusters, which is strongly dominated by Class II and Class III objects.

\section{SUMMARY}

We have identified a high-confidence set of 235 YSOs in Serpens by a set of criteria based on comparison with data from the Spitzer SWIRE Legacy program. This is a large enough number that we can draw important statistical conclusions about various properties of this set. If we assume that the "Class system" of Lada (1987) and Greene et al. (1994) represents an evolutionary sequence, then the relative numbers of YSOs found in each of the first three classes (I, flat, and II) suggest that the Class II phase lasts substantially longer than the combined total of the Class I and flat phases, based on the overall cloud statistics. The clusters, however, show more YSOs in the Class I and flat phases than in Class II, indicating that they are very young. The majority of YSOs (mostly Class II) are outside the clusters and probably represent a somewhat older epoch of star formation compared to the intense star formation now going on in the clusters. The surface density of YSOs in the clusters exceeds that of the rest of the cloud by factors of 10-20 (Table 6).

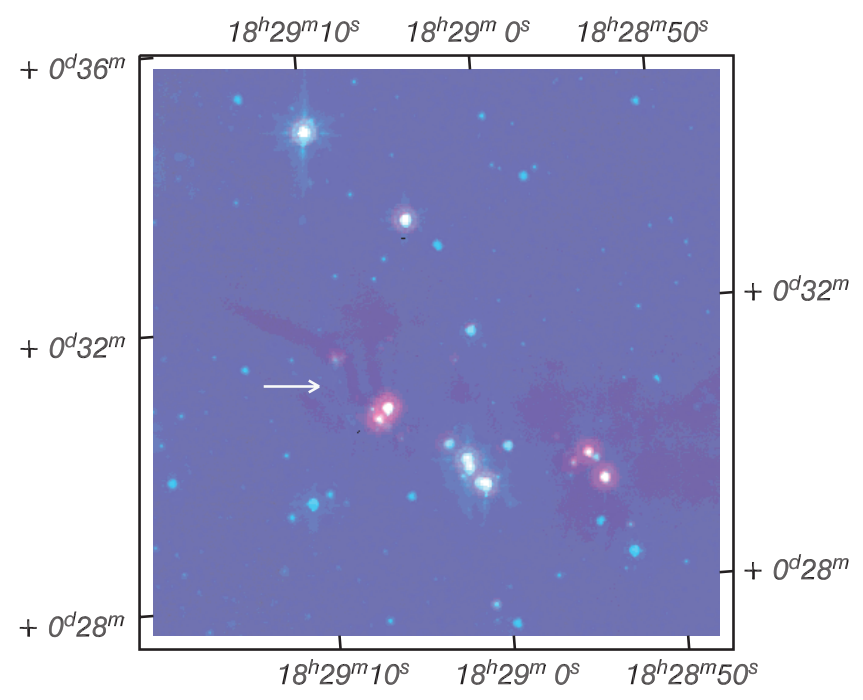

FIG. 24.-Three-color image of the cluster B area of Serpens as for Fig. 23.

The luminosity function for the Serpens YSOs is populated down to luminosities of $3 \times 10^{-3} L_{\odot}$. It may extend even lower, but our ability to distinguish low-luminosity YSOs is severely restricted by the large population of background galaxies at these flux levels. The lower luminosity YSOs, $L<2 \times 10^{-2} L_{\odot}$, exhibit a similar spatial distribution and SED slope distribution to those of their higher luminosity counterparts. This is consistent with the conclusion that they have formed in similar ways.

Support for this work, part of the Spitzer Legacy Science Program, was provided by NASA through contracts 1224608 , 1230782, and 1230779 issued by the Jet Propulsion Laboratory, California Institute of Technology, under NASA contract 1407. Astrochemistry in Leiden is supported by an NWO Spinoza grant and a NOVA grant. B. M. thanks the Fundación Ramón Areces for financial support. This publication makes use of data products from the Two Micron All Sky Survey, which is a joint project of the University of Massachusetts and the Infrared Processing and Analysis Center/California Institute of Technology, funded by NASA and the National Science Foundation. We also acknowledge extensive use of the SIMBAD database.

\section{APPENDIX}

\section{YSO SELECTION PROCESS}

The procedure we use to select YSOs and deselect extragalactic background sources is based on the color-magnitude diagrams shown in Figure 3. We construct "probability" functions for each of the three color-magnitude diagrams based on where a source falls relative to the black dashed lines in each diagram. These three probabilities are multiplied, and then additional adjustments to the probability are made based on several additional properties of the source fluxes and whether or not they were found to be larger than pointlike in the source extraction process.

In the [4.5] versus [4.5] - [8.0] color-magnitude diagram, the probability function is

$$
P_{i 2 i 4}=0.7\left(1-\exp \left\{-\left[1.2+0.5\left(M_{4.5}-D\right)\right]^{3}\right\}\right)
$$

where

$$
D= \begin{cases}13.05, & M_{4.5}-M_{8.0}>1.4 \\ 14.5, & 0.5 \geq M_{4.5}-M_{8.0}<1.4 \\ 12.5, & 0.5 \geq M_{4.5}-M_{8.0}<1.4 \\ 14.5, & M_{4.5}-M_{8.0}<0.5\end{cases}
$$


(for the second value of $D$, the object was found to be pointlike in all IRAC bands, and for the third value of $D$, the object was found to be extended at 3.6 or $4.5 \mu \mathrm{m})$.

In the [24] versus [8.0] - [24] color-magnitude diagram, the probability function is

$$
P_{i 4 m 1}=\exp \left(-\left\{\left[\left(M_{8}-M_{24}-3.5\right) / 1.7\right]^{2}+\left[\left(M_{24}-9\right) / 2\right]\right\}^{2}\right),
$$

and $P_{i 4 m 1}$ is set to a minimum of 0.1 .

In the [24] versus $[4.5]-[8.0]$ color-magnitude diagram, the probability function is

$$
P_{i 24 m 1}=0.7\left(1-\exp \left\{-\left[M_{4.5}-M_{8}+0.8-\left(10-M_{24}\right) / 1.8\right]\right\}\right),
$$

and $P_{i 24 m 1}$ is set to a minimum of 0 .

The combined probability is then

$$
P_{\text {tot }}=P_{i 2 i 4} P_{i 4 m 1} P_{i 24 m 1} \text {. }
$$

In addition, the following factors influence modifications to $P_{\text {tot }}: P_{\text {tot }} /(K-[4.5]), P_{\text {tot }} \times 2$ for sources that are extended at 3.6 or $4.5 \mu \mathrm{m}$, $P_{\text {tot }}=0.1$ where [24] $>10$ (i.e., assumed GALc), and $P_{\text {tot }} \times 0.1$ where $F_{70}>400 \mathrm{mJy}$ and source detected at 5.8, 8, and $24 \mu \mathrm{m}$.

Based on the distribution of $P_{\text {tot }}$ shown in Figure 4, we chose the dividing line between YSOs and extragalactic sources to be $\log _{10} P_{\text {tot }}=-1.47$.

Alcalá, J., et al. 2007, ApJ, submitted

Allen, L. E., et al. 2004, ApJS, 154, 363

2007, in Protostars and Planets V, ed. B. Reipurth, D. Jewitt, \& K. Keil

(Tucson: Univ. Arizona Press), 361

Baraffe, I., Chabrier, G., Allard, F., \& Hauschildt, P. H. 2002, A\&A, 382, 563

Bate, M. R., Clarke, C. J., \& McCaughrean, M. J. 1998, MNRAS, 297, 1163

Cambrésy, L. 1999, A\&A, 345, 965

Casali, M. M., Eiroa, C., \& Duncan, W. D. 1993, A\&A, 275, 195

Chen, C. H., et al. 2005, ApJ, 634, 1372

Cieza, L., et al. 2005, ApJ, 635, 422 2007, ApJ, in press

D’Alessio, P., Calvet, N., Hartmann, L., Lizano, S., \& Canto, J. 1999, ApJ, 527, 893

Davis, C. J., Matthews, H. E., Ray, T. P., Dent, W. R. F., \& Richer, J. S. 1999, MNRAS, 309, 141

Djupvik, A. A., André, Ph., Bontemps, S., Motte, F., Olofsson, G., Galfalk, M., \& Floren, H.-G. 2006, A\&A, 458, 789

Eiroa, C., \& Casali, M. M. 1992, A\&A, 262, 468

Eiroa, C., Djupvik, A. A., \& Casali, M. M. 2007, in ASP Conf. Ser., Handbook of Star Forming Regions, ed. B. Reipurth (San Francisco: ASP), in press

Elmegreen, B. G., Efremov, Y., Pudritz, R. E., \& Zinnecker, H. 2000, in Protostars and Planets IV, ed. V. Mannings, A. P. Boss, \& S. S. Russell (Tucson: Univ. Arizona Press), 179

Enoch, M. L., et al. 2006a, ApJ, 638, 293 2006b, ApJ, submitted

Evans, N. J., II, et al. 2003, PASP, 115, 965 2007, Final Delivery of Data from the c2d Legacy Project: IRAC and MIPS (Pasadena: SSC)

Gomez, M., \& Lada, C. J. 1998, AJ, 115, 1524

Greene, T. P., Wilking, B. A., André, P., Young, E. T., \& Lada, C. J. 1994, ApJ, 434,614

Hartmann, L., Megeath, S. T., Allen, L., Luhman, K., Calvet, N., D’Alessio, P., Franco-Hernandez, R., \& Fazio, G. 2005, ApJ, 629, 881

Harvey, P. M., Wilking, B. A., \& Joy, M. 1984, ApJ, 278, 156

Harvey, P. M., et al. 2006, ApJ, 644, 307 (Paper II) 2007, ApJ, 663, 1139

Hauschildt, P. H., Allard, F., Ferguson, J., Baron, E., \& Alexander, D. R. 1999, ApJ, 525, 871

Hillenbrand, L. A., \& White, R. J. 2004, ApJ, 604, 741
REFERENCES

Hogerheijde, M. R., van Dishoeck, E. F., \& Salverda, J. M. 1999, ApJ, 513, 350 Hora, J. L., Deutsch, L. K., Hoffmann, W. F., \& Fazio, G. G. 1996, AJ, 112, 2064

Horrobin, M. J., Casali, M. M., \& Eiroa, C. 1997, A\&A, 320, L41

Hurt, R. L., \& Barsony, M. 1996, ApJ, 460, L45

Johnstone, D., Wilson, C. D., Moriarty-Schieven, G., Joncas, G., Smith, G., Gregersen, E., \& Fich, M. 2000, ApJ, 545, 327

Jorgensen, J. K., et al. 2006, ApJ, 645, 1246

Kaas, A. A., et al. 2004, A\&A, 421, 623

Kenyon, S. J., \& Hartmann, L. 1987, ApJ, 323, 714

Lada, C. J. 1987, in IAU Symp. 115, Star-forming Regions, ed. M. Peimbert \& J. Jugaku (Dordrecht: Reidel), 1

2006, ApJ, 640, L63

Lada, C. J., et al. 2006, AJ, 131, 1574

Lodieu, N., Caux, E., Monin, J.-L., \& Klotz, A. 2002, A\&A, 383, L15

Maddox, S. J., Efstathiou, G., Sutherland, W. J., \& Loveday, J. 1990, MNRAS, $242,43 \mathrm{P}$

Noreiga-Crespo, A., et al. 2004, ApJS, 154, 352

Padgett, D. L., et al. 2004, ApJS, 154, 433

Preibisch, T. 2003, A\&A, 410, 951

Rebull, L., et al. 2007, ApJS, in press

Robitaille, T. P., Whitney, B. A., Indebetouw, R., Wood, K., \& Denzmore, P. 2006, ApJS, 167, 256

Simon, M. 1997, ApJ, 482, L81

Stassun, K. G., Mathieu, R. D., Luiz, P. R. V., Stroud, N., \& Vrba, F. J. 2004, ApJS, 151, 357

Straizys, V., Cernis, K., \& Bartasiute, S. 1996, Baltic Astron., 5, 125

Strom, S. E., Vrba, F., \& Strom, K. M. 1976, AJ, 81, 314

Surace, J. A., et al. 2004, The SWIRE ELAIS N1 Image Atlases and Source Catalogs (Pasadena: SSC)

Testi, L., \& Sargent, A. L. 1998, ApJ, 508, L91

Testi, L., Sargent, A. L., Olmi, L., \& Onello, J. S. 2000, ApJ, 540, L53

Trams, N. R., et al. 1999, A\&A, 346, 843

van Loon, J. Th., et al. 1999, A\&A, 351, 559

Weingartner, J. C., \& Draine, B. T. 2001, ApJ, 548, 296

Whitney, B. A., Wood, K., Bjorkman, J. E., \& Cohen, M. 2003, ApJ, 598, 1079

Zhang, C. Y., Laureijs, R. J., \& Clark, F. O. 1988a, A\&A, 196, 236

Zhang, C. Y., et al. 1988b, A\&A, 199, 170

Ziener, R., \& Eisloffel, J. 1999, A\&A, 347, 565 